\title{
Chemical Distance Measurement and System Pharmacology Approach Uncover the Novel Protective Effects of Biotransformed Ginsenoside C-Mc against UVB-Irradiated Photoaging
}

\author{
Xiao-yi Liu, ${ }^{1,2,3}$ Hui Li, ${ }^{1}$ Eunson Hwang, ${ }^{3}$ Bom Park, ${ }^{3}$ Yong-kun Xiao, ${ }^{3}$ Senmiao Liu, ${ }^{2}$ \\ Jiansong Fang, ${ }^{4}$ Yeon-Ju Kim $\left(\mathbb{0},{ }^{3}\right.$ Tae-Hoo Yi $\mathbb{1},{ }^{3}$ and Chuipu Cai $\mathbb{D}^{2,4}$ \\ ${ }^{1}$ School of Basic Medical Sciences, Guangzhou University of Chinese Medicine, Guangzhou, China \\ ${ }^{2}$ Division of Data Intelligence, Department of Computer Science, Key Laboratory of Intelligent Manufacturing Technology of \\ Ministry of Education, College of Engineering, Shantou University, Shantou, China \\ ${ }^{3}$ Department of Oriental Medicinal Biotechnology, College of Life Sciences, Kyung Hee University, Republic of Korea \\ ${ }^{4}$ Science and Technology Innovation Center, Guangzhou University of Chinese Medicine, Guangzhou, China
}

Correspondence should be addressed to Yeon-Ju Kim; yeonjukim@khu.ac.kr, Tae-Hoo Yi; drhoo@khu.ac.kr, and Chuipu Cai; chpcai@stu.edu.cn

Received 31 October 2021; Revised 28 November 2021; Accepted 19 January 2022; Published 9 February 2022

Academic Editor: Alin Ciobica

Copyright (C) 2022 Xiao-yi Liu et al. This is an open access article distributed under the Creative Commons Attribution License, which permits unrestricted use, distribution, and reproduction in any medium, provided the original work is properly cited.

Long-term exposure to ultraviolet light induces photoaging and may eventually increase the risk of skin carcinogenesis. Rare minor ginsenosides isolating from traditional medicine Panax (ginseng) have shown biomedical efficacy as antioxidation and antiphotodamage agents. However, due to the difficulty of component extraction and wide variety of ginsenoside, the identification of active antiphotoaging ginsenoside remains a huge challenge. In this study, we proposed a novel in silico approach to identify potential compound against photoaging from 82 ginsenosides. Specifically, we calculated the shortest distance between unknown and known antiphotoaging ginsenoside set in the chemical space and applied chemical structure similarity assessment, drug-likeness screening, and ADMET evaluation for the candidates. We highlighted three rare minor ginsenosides (C-Mc, Mx, and F2) that possess high potential as antiphotoaging agents. Among them, C-Mc deriving from American ginseng (Panax quinquefolius L.) was validated by wet-lab experimental assays and showed significant antioxidant and cytoprotective activity against UVB-induced photodamage in human dermal fibroblasts. Furthermore, system pharmacology analysis was conducted to explore the therapeutic targets and molecular mechanisms through integrating global drug-target network, high quality photoaging-related gene profile from multiomics data, and skin tissue-specific expression protein network. In combination with in vitro assays, we found that C-Mc suppressed MMP production through regulating the MAPK/AP-1/NF- $\kappa$ B pathway and expedited collagen synthesis via the TGF- $\beta /$ Smad pathway, as well as enhanced the expression of Nrf2/ARE to hold a balance of endogenous oxidation. Overall, this study offers an effective drug discovery framework combining in silico prediction and in vitro validation, uncovering that ginsenoside C-Mc has potential antiphotoaging properties and might be a novel natural agent for use in oral drug, skincare products, or functional food.

\section{Introduction}

Cutaneous aging is a multisystem degenerative process characterized by diverse alterations in physiological properties that caused by intrinsic and extrinsic factors. Intrinsic aging occurs as a consequence of hereditary physiological adaptations, and extrinsic aging is mediated by exterior influences such as environmental factors, ultraviolet (UV) exposure, excessive alcohol, and immoderate repetitive muscle use [1, 2]. UV irradiation is the dominant factor in extrinsic aging and can result in hyperpigmentation, wrinkled skin, increased roughness, and loss of elasticity [3]. Previous studies indicated that chronic exposure to UVB radiation could induce skin damage by increasing the levels of reactive 
oxygen species (ROS) and eventually cause characteristic symptoms of skin photoaging, such as hyperplasia of sebaceous glands, deeper and wider wrinkle formation, and reduction in skin elasticity [4-7]. Moreover, there is clear evidence that the development of benign and malignant neoplasms is increased on photoaged skin [8]. Thus, it is a need to develop novel and effective agents against photoaging.

Medicinal Panax (ginseng) herbs, such as Asian ginseng (Panax ginseng C.A. Meyer) and American ginseng (Panax quinquefolius L.), are always the top-selling natural health products that have been promoted as a panacea or "cureall" [9]. As traditional herbal medicine, Panax has a longterm clinical application in dermatological diseases and been mechanistically investigated for its therapeutic effects [10]. Specifically, ginsenosides are the principal constituents responsible for the biological activities of Panax and have been approved for use in various active pharmaceuticals due to their immunoregulatory, anti-inflammatory, antitumor, antiaging, and skin whitening effects [11-14]. Ginsenosides are triterpenoid saponin groups that are classified as dammarane type and oleanane type according to the aglycone skeleton. The dammarane type ginsenosides can be further divided into two groups: PPD-type ginsenosides and protopanaxatriol type ginsenosides [15]. Additionally, previous studies have shown that pharmacological activity is related to the modified glycosylated chain and increases with a reduction in the number of sugar moieties [16]. Recently, the rare minor ginsenosides have been proved to be more efficient active pharmacological agents compared with the major ginsenosides [17]. Accordingly, many rare ginsenosides such as $\operatorname{Rg} 3$ and C-Mx have been demonstrated pharmacological activities with regard to UVB-induced skin aging [18, 19]. Until now, hundreds of rare ginsenosides have been identified. However, most of them are extremely difficult to extract or efficient produce. Therefore, considering the labor and time cost, it is inevitable a huge challenge to comprehensively evaluate the protective effect against photoaging of these ginsenosides via conventional experimental assays.

In recent years, in silico approaches have been broadly applied in drug discovery and successfully identified novel drug candidates for the prevention and treatment of many diseases [20-22]. Ligand-based methods, such as quantitative structure-activity relationship (QSAR) models constructed by machine learning or deep learning algorithm, are the most common used approaches [23-25]. However, these approaches require high quality of training set containing sufficient and diverse samples, which is not suitable for the drug discovery of photoaging owing to the lack of enough experimental and medicinal data. Structure-based approaches such as molecular docking are always encumbered by the computing speed and the resolution of crystal structures [23]. Besides, network approach based on system pharmacology, which comprehensively considers the information of drug-target network and disease-related genes, provides new insights into the identification of active ingredients against disease [26-28]. Nevertheless, as the object of this study is rare ginsenosides, most of which remain nearly unknown for the information of interacted targets. Since current approaches could not be adequate for the requirements, it is necessary to design a novel efficient in silico strategy to identify the potential active agents for photodamage from various ginsenosides.

In this study, we proposed an in silico and in vitro integrated framework for identification of active ginsenosides against photoaging (Figure 1 and Supplementary Figure S1). Specifically, we first designed a novel drug virtual screening protocol, which consists of shortest distance measurement in the chemical space of principle component analysis, pairwise chemical structure similarity analysis, drug-likeness screening, and ADMET (adsorption, distribution, metabolism, excretion, and toxicity) evaluation (Figure 1(a)). Based on the computational prediction, the rare minor ginsenoside $\mathrm{C}-\mathrm{Mc}$ was selected to evaluate its protective effect towards UVB-irradiated photodamage in human skin dermal fibroblasts (Figure 1(b)). After that, system pharmacology-based analysis, including construction of integrated network, gene enrichment analysis, and functional module induction, was performed to explore the potential therapeutic targets, biological process, signal pathway, and regulatory function (Figure 1(c)). Finally, further in vitro experiments were conduct to validate the predictions and systematically elucidate the antiphotoaging molecular mechanism of C-Mc (Figure 1(d)).

\section{Materials and Methods}

\subsection{In Silico Experiments}

2.1.1. Collection of Ginsenosides. The information and structures of ginsenosides were collected from literatures and PubChem database of National Center for Biotechnology Information (NCBI). These compounds were further converted to unified InChiKey and Smiles format using Openbabel tool [29]. Totally, 98 ginsenosides with unique structure were obtained. Through consulting literature materials, 16 of them with previous reported antiphotoaging or photoprotective effects are labeled as known antiphotoaging agents, while the remaining 82 compounds were selected as unknown objects for screening (Supplementary Table S1).

\subsubsection{Molecular Processing and Principal Component} Analysis (PCA). The collected ginsenosides were processed by molecular washing and energy minimizing for protonating strong bases, deprotonating strong acids, removing inorganic counter ions, adding hydrogen atoms, and generating stereo isomers and valid single 3D conformers using MOE 2010 [30]. Furthermore, two-dimensional (2D) descriptors were generated by MOE 2010 to represent molecular properties and structural information. The MOE descriptor set consists of 186 features, covering physical property, pharmacophore feature, atom count and bond count, adjacency and distance matrix, subdivided surface area, Kier and Hall connectivity and Kappa shape indices, and partial charge descriptors. After that, principal component analysis (PCA) was performed by MOE to reduce the dimensionality 


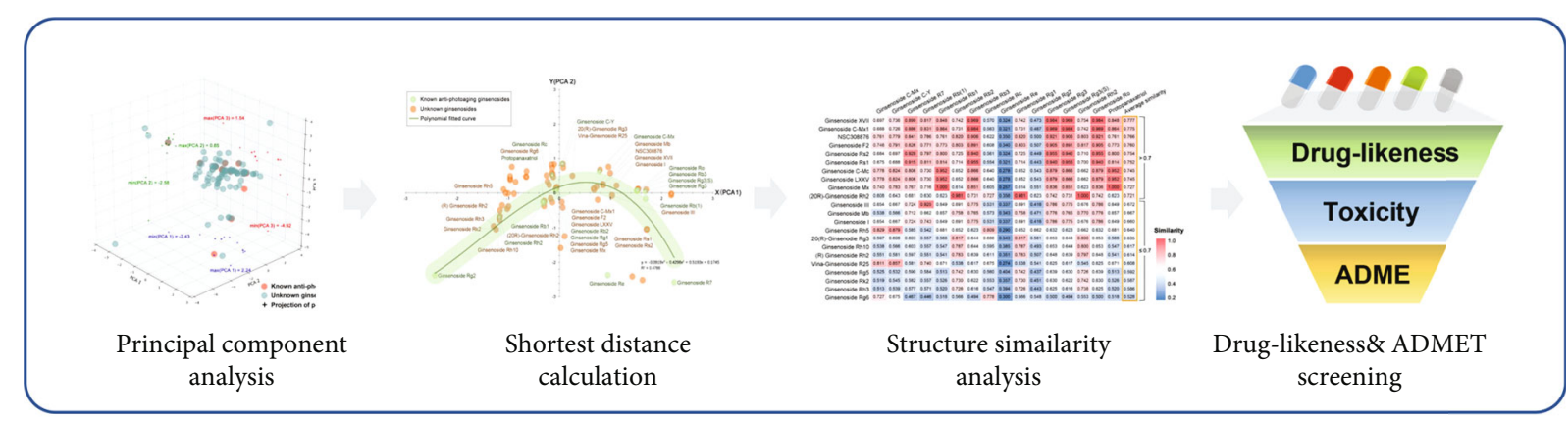

(a)

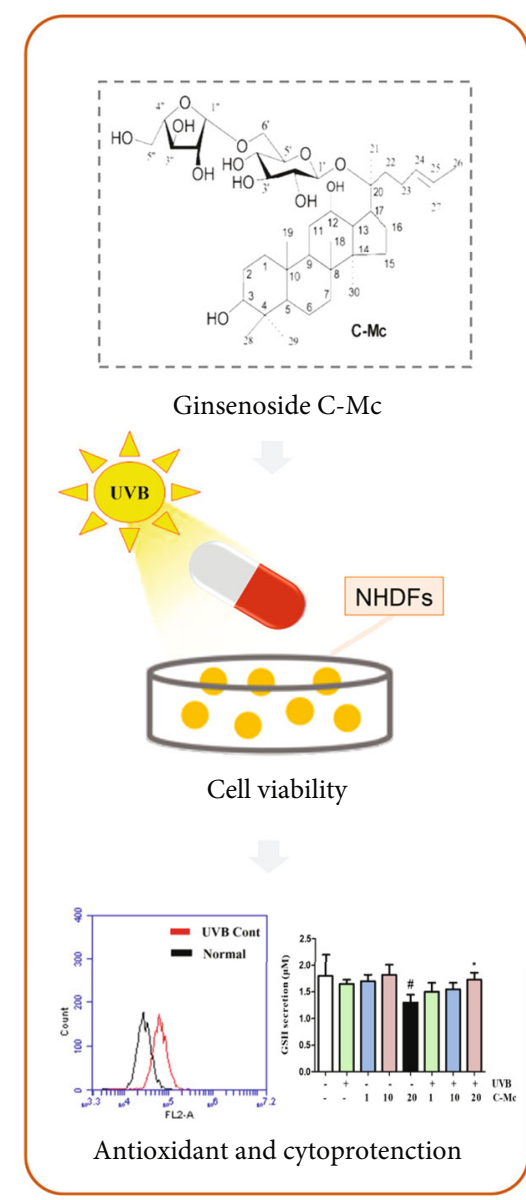

(b)

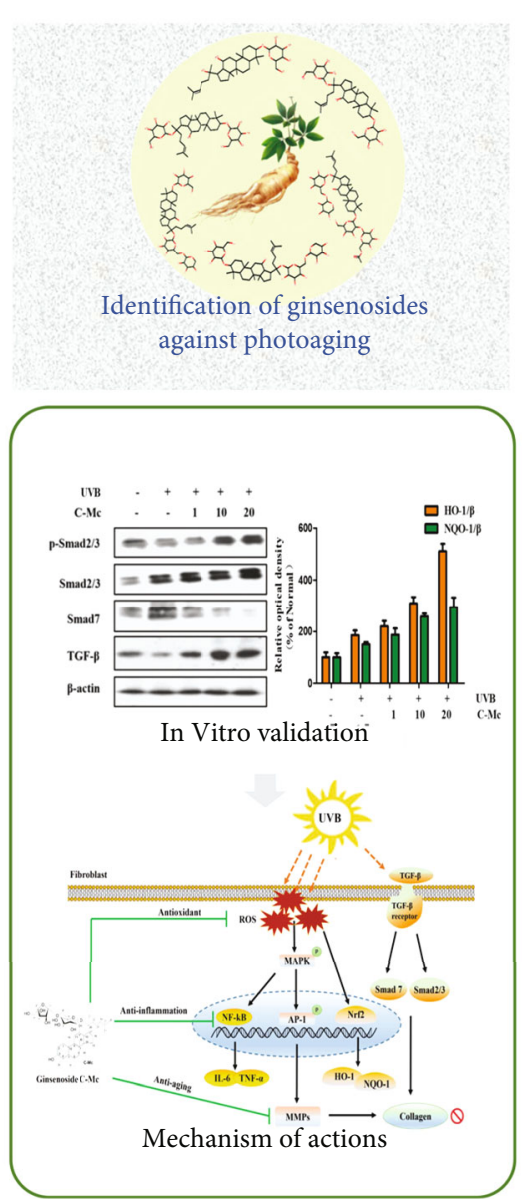

(d)

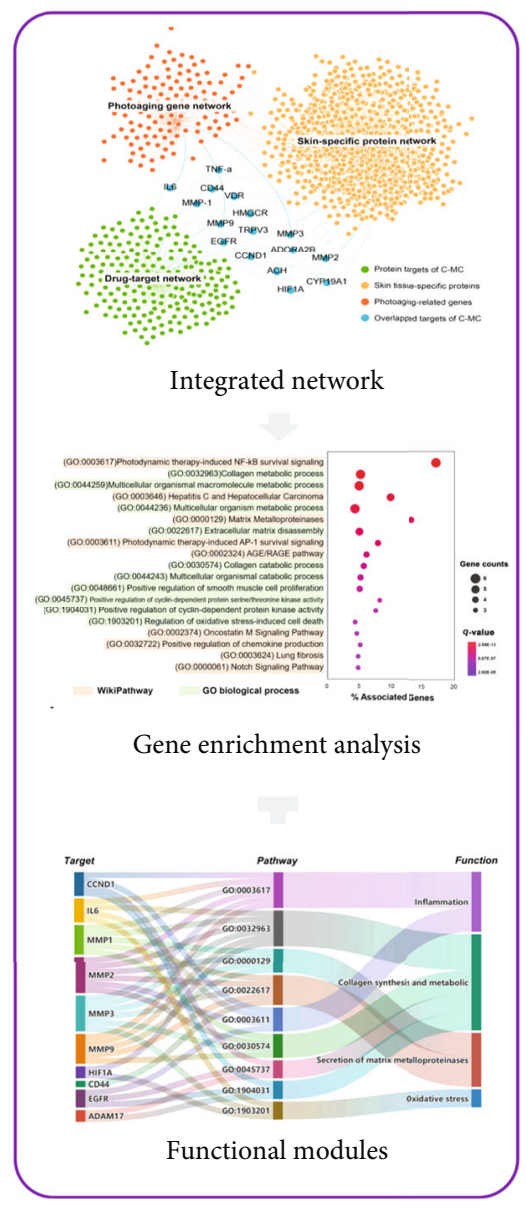

(c)

FIGURE 1: Schematic diagram illustrating in silico methodology and in vitro validation for identification of active ginsenosides against photoaging. (a) In silico identification of antiphotoaging candidates from 82 ginsenosides. (b) In vitro validation of antiphotoaging effects for the promising candidate ginsenoside C-Mc. (c) System pharmacology-based exploration of the potential therapeutic targets, biological process, signal pathway, and regulatory function. (d) In vitro assay for elucidating the mechanism actions of ginsenoside C-Mc against photoaging.

of molecular descriptors by linearly transforming the data. The 186 molecular descriptors were used as input variables. The detailed information of the PCA computational procedure can be found in the following references $[30,31]$.

\subsubsection{Shortest Distance Measurement among Compounds in} the PCA Space. The first two outputs of PCA for each ginsenoside were defined as its position in the two-dimensional
PCA space. Each point in the coordinate represents a ginsenoside. We hypothesize that a ginsenoside without previous antiphotoaging reports exhibits a high possibility to possess photoprotective effects if its position in the PCA space is relatively close to those of the known antiphotoaging ginsenosides. Here, the points of the 16 known antiphotoaging ginsenosides were first fitted as a curve by a polynomial. Then, the shortest distance $(d)$ between each point of 
unknown ginsenoside $Q\left(x_{0}, y_{0}\right)$ and each point on the fitted curve of the known ginsenosides $f(x, y)=0$ was calculated, as the Euclidean distance metric described below:

$$
d=\sqrt{\left(x-x_{0}\right)^{2}+\left(y-y_{0}\right)^{2}}
$$

Demand that

$$
L(x, y)=\left(x-x_{0}\right)^{2}+\left(y-y_{0}\right)^{2}+\gamma f(x, y)
$$

The following equations could be obtained by Lagrange multipliers:

$$
\left\{\begin{array}{l}
L_{x}(x, y)=2\left(x-x_{0}\right)+\gamma f_{x}(x, y)=0 \\
L_{y}(x, y)=2\left(y-y_{0}\right)+\gamma f_{y}(x, y)=0 \\
f(x, y)=0
\end{array}\right.
$$

where $f x(x, y)$ and $f y(x, y)$ are the partial derivatives of $f($ $x, y)$ with respect to $x$ and $y$. By applying equation set (3), the corresponding point $P(x, y)$ on the curve is obtained, and the shortest distance $d_{\text {min }}$ between $P$ and $Q$ can be calculated by the Euclidean distance Equation (1). Assume the endpoints of curve are $M$ and $N$, the shortest distance $D_{\text {min }}$ between point $Q$ and the fitted curve is the minimum value among $d_{\min }(Q, P), d_{\min }(Q, M)$, and $d_{\min }(Q, N)$, which can be described as follows:

$$
D_{\min }=\min \left\{\begin{array}{l}
d_{\min }(Q, P), \\
d_{\min }(Q, M), \\
d_{\min }(Q, N)
\end{array}\right.
$$

In this study, according to the domain of chemical space, the shortest distance smaller than 0.2 is considered as significant.

2.1.4. Pairwise Chemical Structure Similarity Analysis. In addition to the PCA based on chemical molecular properties, the chemical similarity analysis based on chemical structures was also applied to measure the distance between each pair of known and unknown antiphotoaging ginsenosides. The fp_topological_4 fingerprint was selected to represent the structures of ginsenosides, while Tanimoto metric was used to calculate the similarity index between each other, as described below:

$$
\text { Tanimoto similarity }=\frac{c}{(a+b-c)},
$$

where $a$ and $b$ represent the bits (number of fingerprint descriptors) in chemical structure of $\operatorname{Cmp}(m)$ and $\operatorname{Cmp}(n)$ and $c$ refers to the bits coexist in both $\operatorname{Cmp}(m)$ and $\mathrm{Cmp}($ $n)$. A higher Tanimoto index means a higher similarity of their structures.

2.1.5. Drug-Likeness Evaluation and ADMET Screening. In this study, the drug-likeness evaluation and ADMET screen- ing were utilized to further exclude the candidates with undesirable pharmacokinetic properties and unacceptable toxicity. Specifically, the drug-likeness predictive model is provided by Dong's study, which was constructed by the machine learning algorithms of random forest (RF) with molecular descriptors of MACCS on a training set containing 6,731 positive and 6,769 negative samples [32]. Besides, pan assay interference compounds (PAINS), one of the most famous frequent hitters filters, was applied to screen potential false positive hits [33]. The ADMET properties, including human oral bioavailability $20 \%\left(F_{20 \%}\right)$, plasma protein binding (PPB), rat acute oral toxicity (AOT), and skin sensitization, were evaluated by ADMETlab 2.0, a platform that integrated a series of well-performed predictive models for pharmacokinetics and toxicity [34].

2.1.6. Construction of Drug-Target (D-T) Network of Ginsenoside $C-M c$. Since currently there are no reported protein targets regulated by ginsenoside C-Mc, the D-T network was constructed through integrating predicted drugtarget interactions (DTIs) obtained by the following ways: (1) target identification based on molecular docking simulation. The crystal structures of interested protein targets were retrieved from Protein DataBank (PDB), optimized in ChemBioOffice 2010 tool package, and implemented molecular docking with $\mathrm{C}-\mathrm{Mc}$ according to the available standard Autodock protocol $[35,36]$. We found C-Mc showed higher binding affinities to receptors MAPK, NF- $\kappa$ B, IL- 6 , and TNF- $\alpha$ compared with control ligands (Supplementary Figure S2 and Table S2); (2) manually collection of reported targets of other ginsenosides from relevant literatures and our previous integrated DTI database of natural product [37]. It is plausible to hypothesize that these targets are more likely to interact with C-Mc since the ginsenosides are similar in structure. A total of 128 targets were collected; (3) potential target proteins predicted by balanced substructure-drug-target networkbased inference (bSDTNBI) methods, a computational approach that can predict potential targets for new chemical entities [38, 39]. The molecular fingerprint of Klekota-Roth and default parameters were used, and the top 100 predicted targets for each compound were preserved. Finally, the D-T network contains 222 DTIs after removing the duplicated interactions and non-Homo sapiens proteins (Supplementary Table S3).

\subsubsection{Photoaging-Related Gene Profile Integrating from} Multiomics Data. We manually curated and integrated a skin photoaging-related gene set from multiple resources, including (1) PolySearch 2.0, a significantly improved textmining system for discovering associations biomedical entities [38]. The disease name of "photoaging" was inputted as query key word; (2) RNA sequencing data of photodamaged skins from Genotype-Tissue Expression (GTEx) project. The transcriptomic changes induced by UV irradiation were characterized through differentially expressed gene analysis and weighted gene coexpression network analysis [40]; (3) differentially expressed genes screened by microarray analysis between sun-exposed (anterior ear skin) and sun- 
protected (retroauricular skin) skins of 6 patients with facial photoaging [41]; and (4) published skin photoaging-related study literatures from PubMed database (as of May 2021). All the collected proteins/genes were converted into unified Homo sapiens gene symbol name and Entrez ID based on the NCBI Gene Database (https://www.ncbi.nlm.nih.gov/ gene). Finally, 124 unique photoaging-related genes were collected after removing the duplicated ones (Supplementary Table S4).

2.1.8. Skin Tissue-Specific Expression Protein Network. The RNA-seq data (RPKM value) of skin tissue was extracted from the GTEx V6 release (https://gtexportal.org/home/). In this work, genes $(i)$ with RPKM value $\geq 1$ in over $80 \%$ of skin samples were considered as specific expressed in the skin tissue $(t)$. The average expression $E(i)$ and the standard deviation $\delta_{E(i)}$ of a gene's expression towards the skin tissue were calculated, and significance of gene $(i)$ expression in the skin tissue $(t)$ was quantified by the equation given below:

$$
Z_{E(i, t)}=\frac{E(i, t)-E(i)}{\delta_{E(i)}} .
$$

We asserted that genes with $Z$-expression score larger than 2 were regarded as high expressed in the skin tissue. The information of the skin tissue-specific expression protein network containing 907 proteins is provided in Supplementary Table S5.

\subsection{In Vitro Experiments}

2.2.1. Chemicals and Biochemical. Ginsenosides Rc and CMc were obtained from Ambo Laboratory (Dacejeon, Korea). ELISA kits for MMPs (MMP-1 and MMP-3), IL-6, TGF- $\beta 1$, procollagen type I, and VEGF were purchased from R\&D Systems, Inc. (Minneapolis, MN, USA). DMEM, FBS, and penicillin/streptomycin were purchased from Gibco BRL (Aidenbac, Germany). Glutathione content was assayed using a GSH assay kit (Cayman Chemical Co, Ann Arbor, MI, USA), and the lactate dehydrogenase (LDH) cytotoxicity assay kit was purchased from Roche Diagnostics $\mathrm{GmbH}$ (Roche Diagnostics, Mannheim, Germany). Antibodies were purchased from Santa Cruz Biotechnology (Santa Cruz, CA, USA) and Cell Science (Canton, MA, USA). Solvents were purchased from Samchun Pure Chemicals (Korea) unless otherwise stated.

2.2.2. Preparation and Identification of Ginsenoside C-Mc. The minor ginsenoside C-Mc was prepared from American ginseng PPD ginsenosides, using 6\% PPD ginsenoside substrate in acetate buffer (0.02 M and pH 5.0). Ginsenoside $\mathrm{C}-\mathrm{Mc}$ was reacted with a volume of crude enzyme $(A$. niger g.848 strain) in the bioreactor.

In brief, chromatographic analysis was completed by a Waters ACQUITY UPLC system using XBridge C-18 chromatographic column $(5 \mu \mathrm{m}, \varphi 4.6 \times 250 \mathrm{~mm})$ was used to analyze the samples. The mobile phase was acetonitrile (A) and water (B), column oven was $35^{\circ} \mathrm{C}$, and flow rate is $0.6 \mathrm{ml} / \mathrm{min}$. Detection wavelength of the DAD was $203 \mathrm{~nm}$.

The mass examination was completed by a Waters SQ detector through a positive electrospray ionization pattern. Briefly, cone gas flow was set at $50 \mathrm{~L} / \mathrm{h}$, and desolvation gas flow was $550 \mathrm{~L} / \mathrm{h}$. Capillary voltage and cone voltage were $3.5 \mathrm{kV}$ and $30 \mathrm{~V}$, respectively.

The sample of ginsenoside C-Mc was identified as 20-O[ $\alpha$-L-arabinofuranosyl- $(1 \rightarrow 6)-\beta$-D-glucopyranosyl]-20(S)protopanaxdiol using a BrukeAvance $600\left({ }^{1} \mathrm{H}\right.$ : $600 \mathrm{MHz}$ and ${ }^{13} \mathrm{C}$ : $150 \mathrm{MHz}$ ) NMR spectrometer (Switzerland) in our previous study [42].

2.2.3. Cell Culture, UVB Irradiation, and Ginsenoside C-Mc Treatment. Normal human dermal fibroblasts (NHDFs) were obtained from a skin biopsy healthy male donor (MCTT, Seoul, Korea) and grown in DMEM (1\% penicillin-streptomycin and $10 \%$ heat-inactivated FBS) in $5 \% \mathrm{CO}_{2}$ incubator at $37^{\circ} \mathrm{C}$. In brief, experimental groups were exposed to UVB irradiation at $144 \mathrm{~mJ} / \mathrm{cm}^{2}$ using a Bio-Link BLX-312 machine (Vilber Lourmat $\mathrm{GmbH}$, France) and then treated with 1,10 , or $20 \mu \mathrm{M}$ ginsenoside C-Mc. Control groups were subjected to the same protocol without exposure to UVB radiation.

2.2.4. Cell Viability Assay. The effect of ginsenoside C-Mc on viability of NHDFs was evaluated by MTT assay. After treatment for $72 \mathrm{~h}, 1 \mathrm{~mL}$ of medium was removed for ELISA. MTT solution $(100 \mu \mathrm{g} / \mathrm{ml}$ MTT in PBS) was added to the remaining medium followed by incubation at $37^{\circ} \mathrm{C}$ for $4 \mathrm{~h}$. After removal of the medium, $800 \mu \mathrm{L}$ DMSO was added to each well and the absorbance was detected at $595 \mathrm{~nm}$.

2.2.5. Measurement of ROS Scavenging Ability. After UVB irradiation $\left(144 \mathrm{~mJ} / \mathrm{cm}^{2}\right)$, NHDFs were washed twice with PBS and treated with ginsenoside C-Mc $(1,10$, and $20 \mu \mathrm{M})$ for $24 \mathrm{~h}$. The cells were stained with $30 \mathrm{mM} 2^{\prime} 7^{\prime}$-dichlorofluorescein diacetate (DCFH-DA; Sigma-Aldrich) for $30 \mathrm{~min}$ at $37^{\circ} \mathrm{C}$ and then analyzed by flow cytometry (BD Accuri C6; Becton-Dickinson, San Jose, CA, USA).

2.2.6. Intracellular GSH Determination. The level of GSH reductase was tested using a GSH assay kit (Cayman Chemical Co, Ann Arbor, MI). Briefly, the sulfhydryl group of GSH reacts with 5,5' -dithiobis-2-nitrobenzoic acid (DTNB) to yield the product 5-thio-2-nitrobenzoic acid (TNB). The final absorbance was detected at $405 \mathrm{~nm}$.

2.2.7. Production of $M M P-1, I L-6, M M P-3$, Procollagen Type I, TGF- $\beta 1$, and VEGF. The concentrations of MMP-1, IL-6, MMP-3, procollagen type I, TGF- $\beta 1$, and VEGF in cell medium were quantified using commercially available ELISA kits as described above. Each experiment was repeated at least three times.

2.2.8. Lactate Dehydrogenase (LDH) Release. A LDH cytotoxicity assay kit (Roche Diagnostics, Mannheim, Germany) was used to measure the LDH level. In this assay, LDH reduces NAD to NADH, which then interacts with a specific 

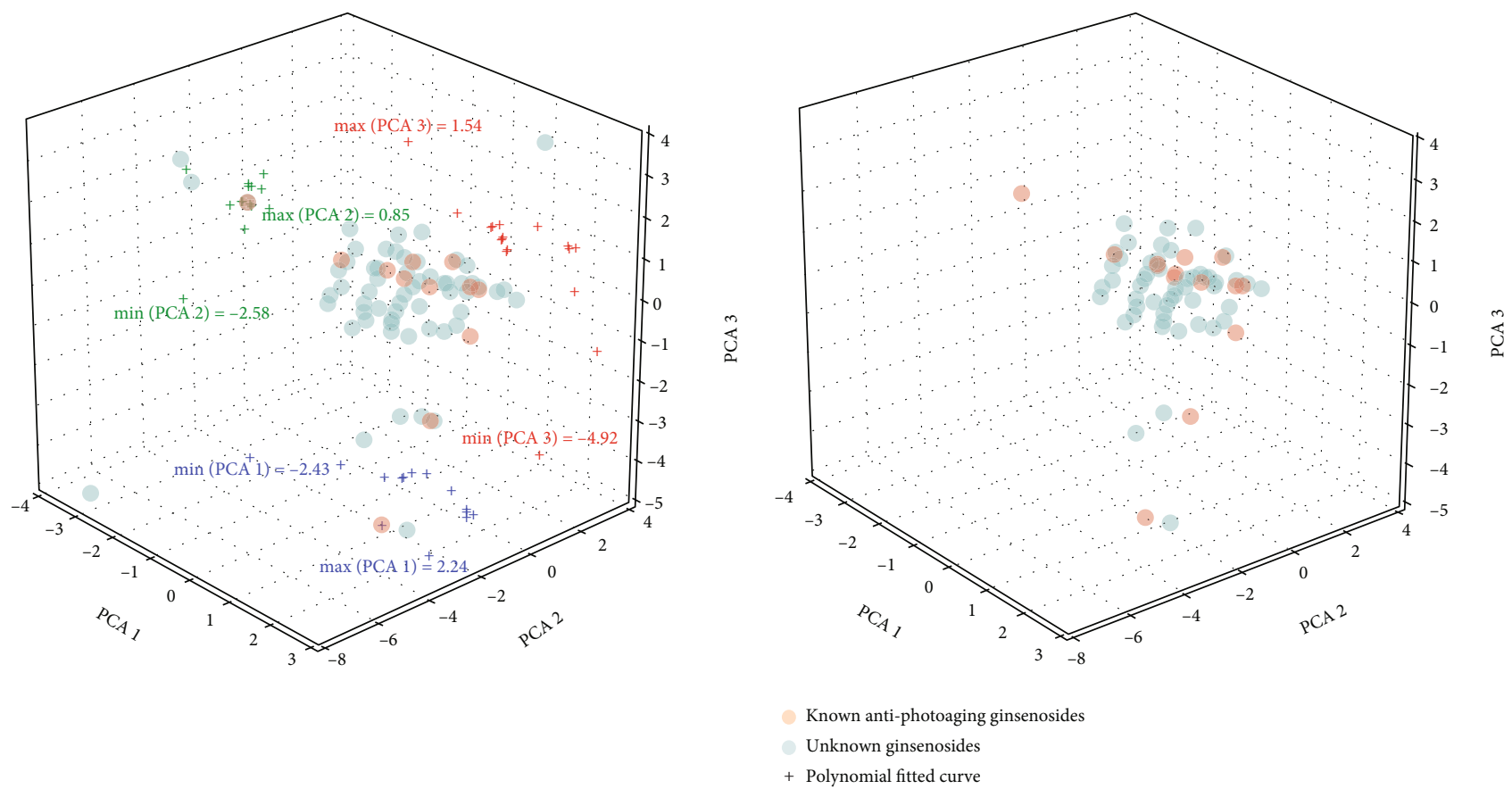

(a)

(b)

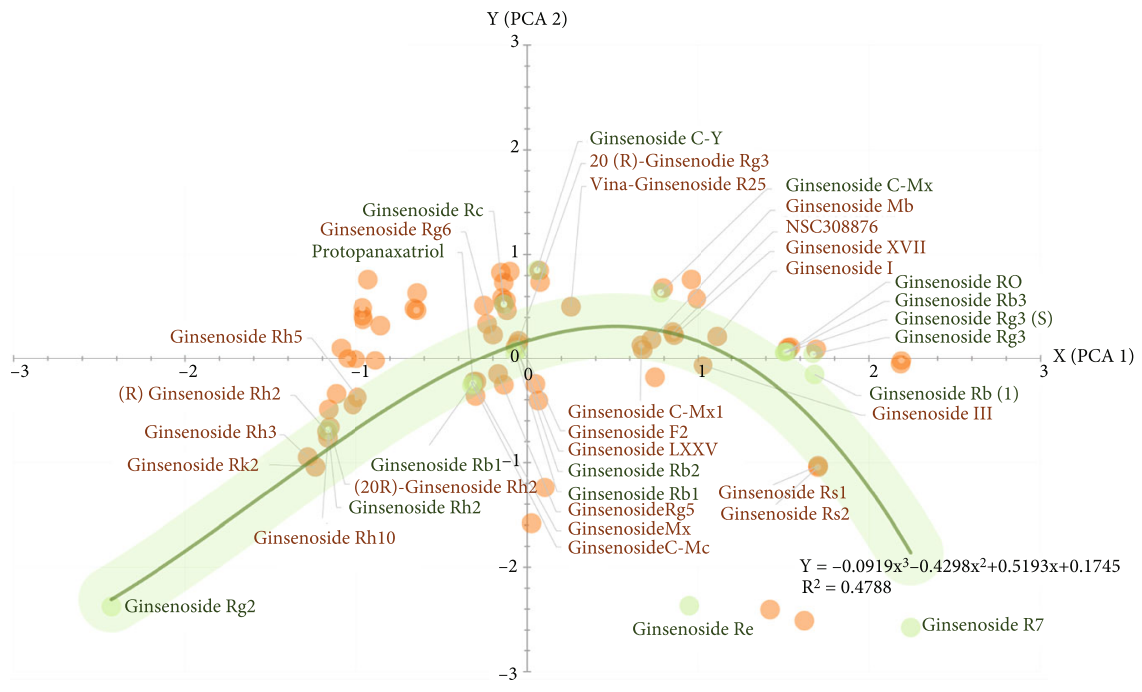

Known anti-photoaging ginsenosides

Unknown ginsenosides

Polynomial fitted curve

(c)

FIGURE 2: Preliminary identification of potential antiphotoaging ginsenosides through shortest distance measurement among compounds in the PCA space. (a) Calculation of the rational boundary constraint of the space of PCA. (b) Eliminating the improper compounds outside the boundary constraint of the space. (c) Shortest distance calculation between known and unknown antiphotoaging ginsenosides.

probe to produce a colored product. After incubation for $40 \mathrm{~min}$ at $37^{\circ} \mathrm{C}$, the absorbance was detected at $450 \mathrm{~nm}$.

2.2.9. Quantitative Real-Time RT-PCR. NHDFs were harvested after UVB irradiation $\left(144 \mathrm{~mJ} / \mathrm{cm}^{2}\right)$ and treated with ginsenoside C-Mc $(1,10$, and $20 \mu \mathrm{M})$. RNA was isolated from cells using TRIZOL reagent according to the manufacturer's instructions (Invitrogen Life Technologies, Carlsbad, CA). The expression of MMP-1, MMP-3, procollagen type I, iNOS, TNF- $\alpha$, and IL- 6 mRNA was measured by real- time PCR using SYBR ${ }^{@}$ Green master mix in a BioRad CFX Connect Real-Time PCR Detection System (BioRad, Hercules, CA). GAPDH was used for internal normalization. All experiments were carried out in triplicate. The primer sequences are provided in Supplementary Table S6.

2.2.10. Cytosolic and Nuclear Extracts. Cells were harvested after UVB irradiation $\left(144 \mathrm{~mJ} / \mathrm{cm}^{2}\right)$, and the protein was extracted. Cytosolic and nuclear fractions were separated 


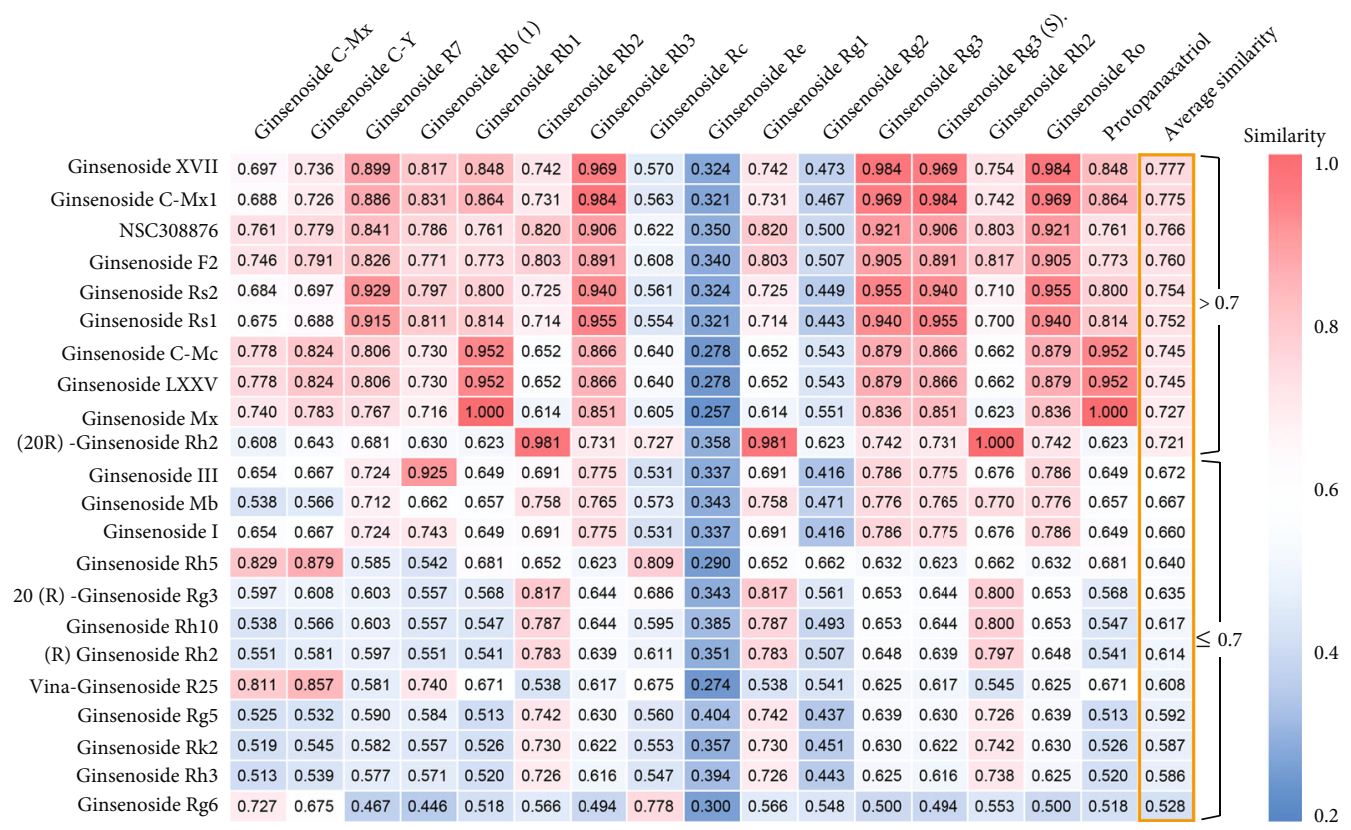

(a)

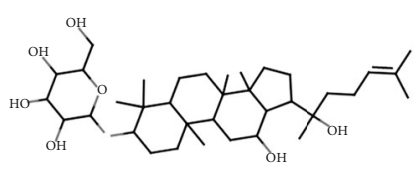

(20R) -Ginsenoside Rh2

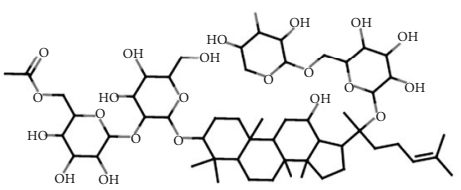

Ginsenoside Rs1

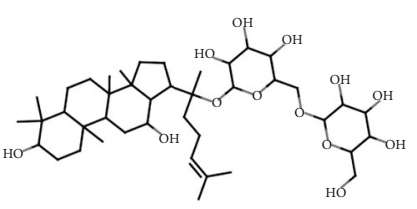

Ginsenoside LXXV

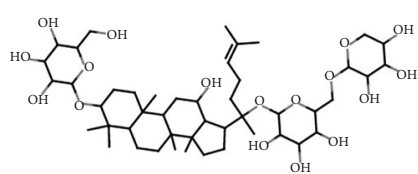

Ginsenoside C-Mxl

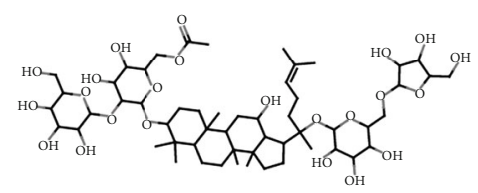

Ginsenoside Rs2

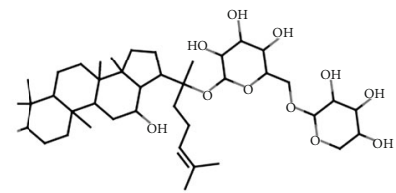

Ginsenoside Mx

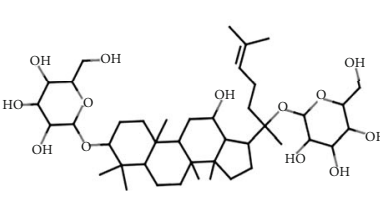

Ginsenoside F2

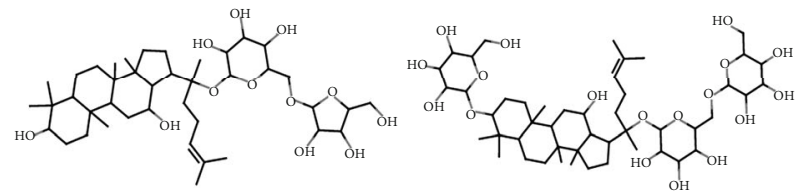

Ginsenoside C-MC

Ginsenoside XVII

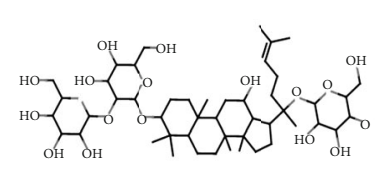

NSC308876

(b)

FIGURE 3: Pairwise chemical structure similarity analysis between known and unknown antiphotoaging ginsenosides. (a) Heatmap illustrating the similarity analysis results of the 16 known active ginsenosides and the 22 unknown ginsenosides. (b) Chemical structures of the top 10 ginsenosides with average similarity index higher than 0.7 .

using a commercial kit (NE-PER nuclear and cytoplasmic extraction reagents; Pierce).

2.2.11. Western Blot Analysis. Western blotting was performed to detect components of the MAPK/AP-1, Nrf2/ ARE, TGF- $\beta /$ Smad, and NF- $\kappa \mathrm{B} / \mathrm{I} \kappa \mathrm{B}-\alpha$ signaling pathways. In brief, cells were lysed with RIPA buffer (Cell Signaling Technology, USA) and centrifuged. Protein concentration was measured using Bradford reagent, and equal amounts of protein for each sample were separated by $8 \%$ or $10 \%$ SDS-PAGE and subjected to immunoblotting using a standard protocol and an ECL Western blot detection system (Amersham Pharmacia Biotech, NJ, USA).

2.3. Statistical Analysis. All data were based on three independent experiments. Data were expressed as mean \pm SD using GraphPad Prism 5 (GraphPad Software, Inc., CA, USA). Statistical comparisons between different treatments 
TABLe 1: Drug-likeness evaluation and ADMET screening for the 10 predicted antiphotoaging ginsenosides.

\begin{tabular}{|c|c|c|c|c|c|c|}
\hline Drug & Drug-likeness & PAINS & $F_{20 \%}$ & $\mathrm{PPB}$ & $\mathrm{AOT}$ & Skin sensitization \\
\hline Ginsenoside XVII & 0.848 & 0 & 0.986 & $72.66 \%$ & 0 & 0.005 \\
\hline Ginsenoside C-Mx1 & 0.854 & 0 & 0.981 & $75.10 \%$ & 0 & 0.008 \\
\hline NSC308876 & 0.856 & 0 & 0.986 & $72.90 \%$ & 0 & 0.005 \\
\hline Ginsenoside F2 & 0.846 & 0 & 0.67 & $83.91 \%$ & 0 & 0.015 \\
\hline Ginsenoside Rs2 & 0.812 & 0 & 0.996 & $60.39 \%$ & 0 & 0.002 \\
\hline Ginsenoside Rs1 & 0.804 & 0 & 0.995 & $60.82 \%$ & 0 & 0.002 \\
\hline Ginsenoside C-Mc & 0.818 & 0 & 0.608 & $86.59 \%$ & 0 & 0.034 \\
\hline Ginsenoside LXXV & 0.818 & 0 & 0.753 & $83.36 \%$ & 0 & 0.023 \\
\hline Ginsenoside $M x$ & 0.764 & 0 & 0.655 & $86.74 \%$ & 0 & 0.032 \\
\hline (20R)-Ginsenoside Rh2 & 0.772 & 0 & 0.076 & $94.05 \%$ & 0 & 0.038 \\
\hline
\end{tabular}

Note: PAINS: pan assay interference compounds; $F_{20 \%}$ : human oral bioavailability $20 \%$; PPB: plasma protein binding; AOT: rat acute oral toxicity. The value of drug-likeness refers to the probability of being positive. The value of PAINS refers to the number of alert substructures; PPB with predicted value $<90 \%$ is considered proper. The values of $F_{20 \%}$, AOT, and skin sensitization are the probabilities of being $F_{20 \%+}$ (bioavailability $<20 \%$ ), toxicity $(<500 \mathrm{mg} / \mathrm{kg}$ ), and sensitizer, while probability higher than 0.7 represents a poor result according to the explanation of ADMETlab 2.0 [34].

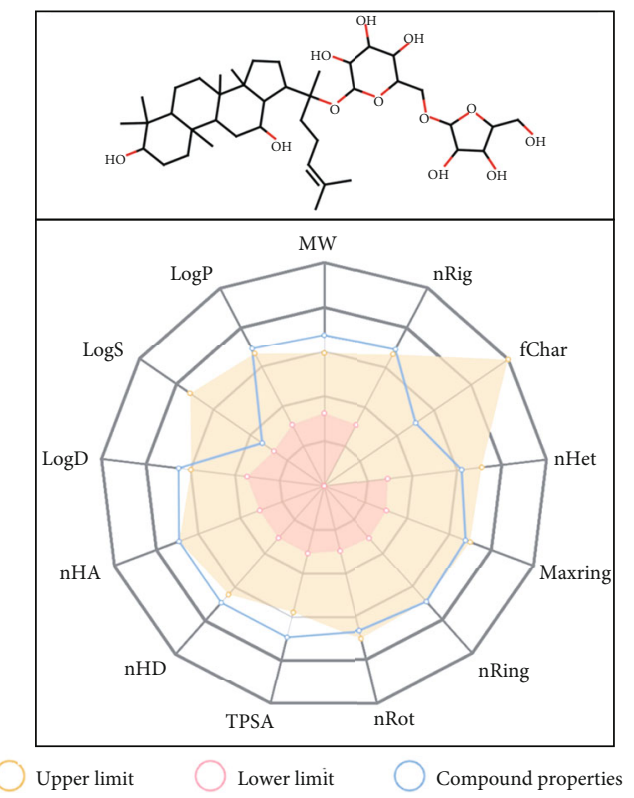

(a)

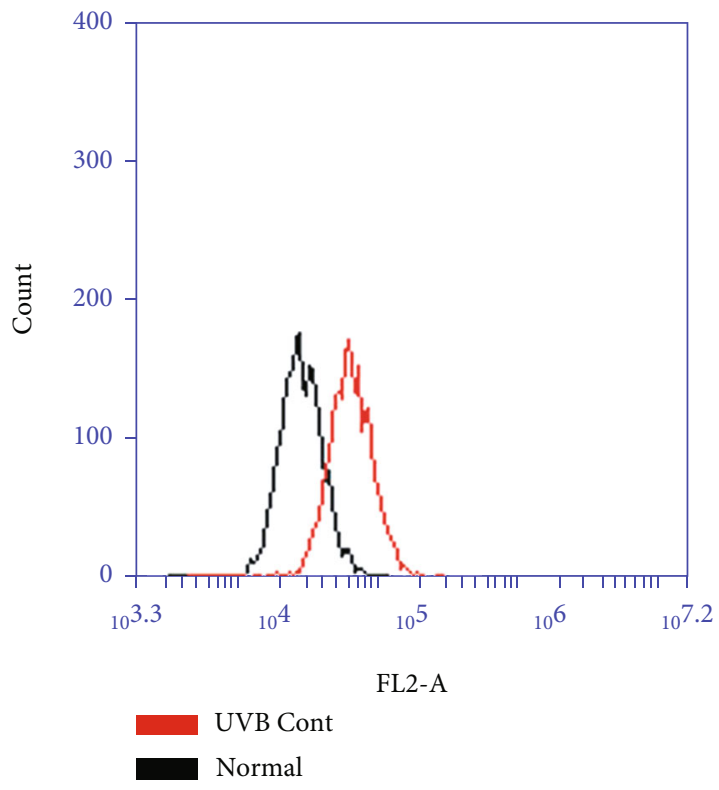

(b)

FIgURE 4: Continued. 

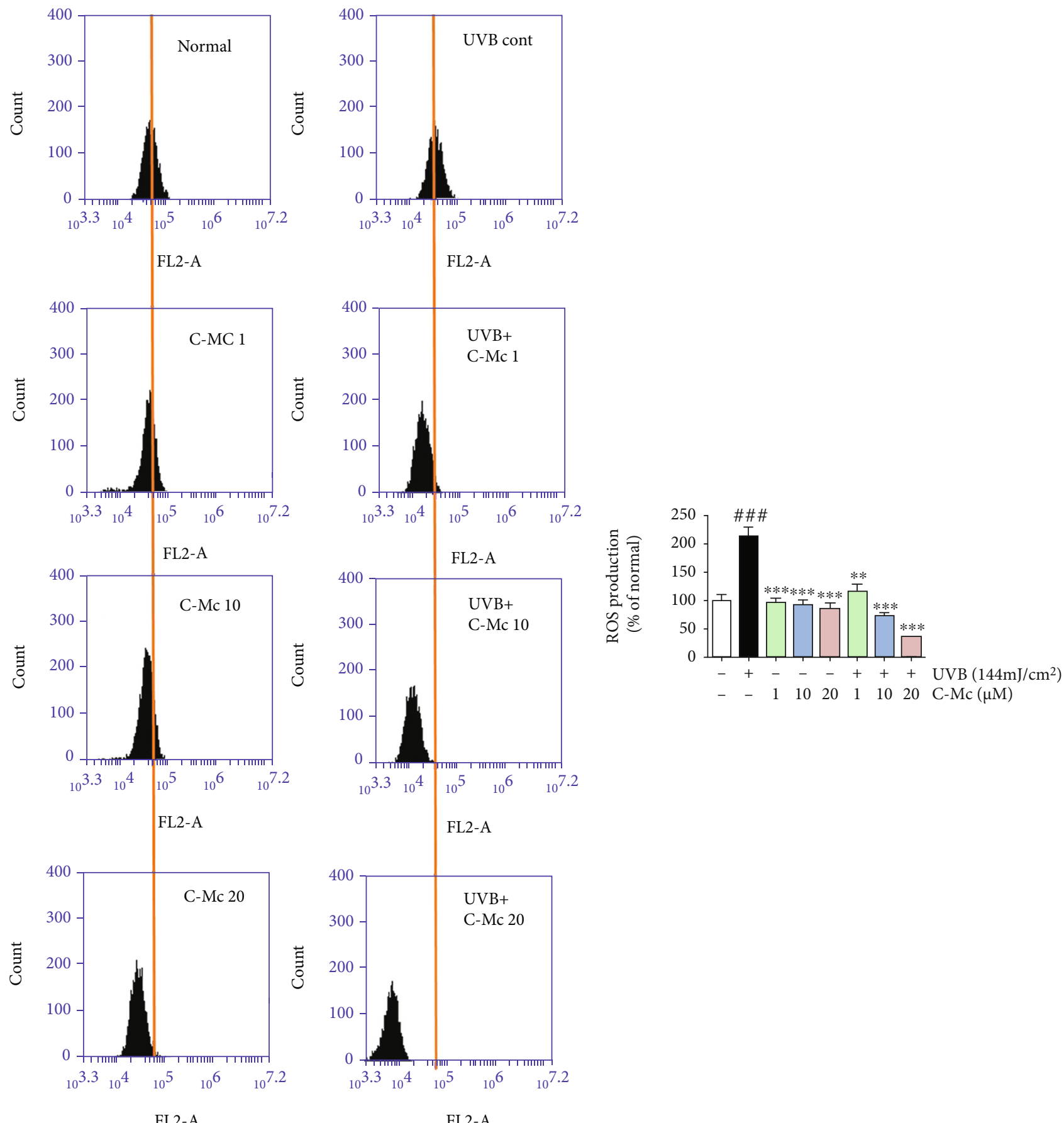

(c)
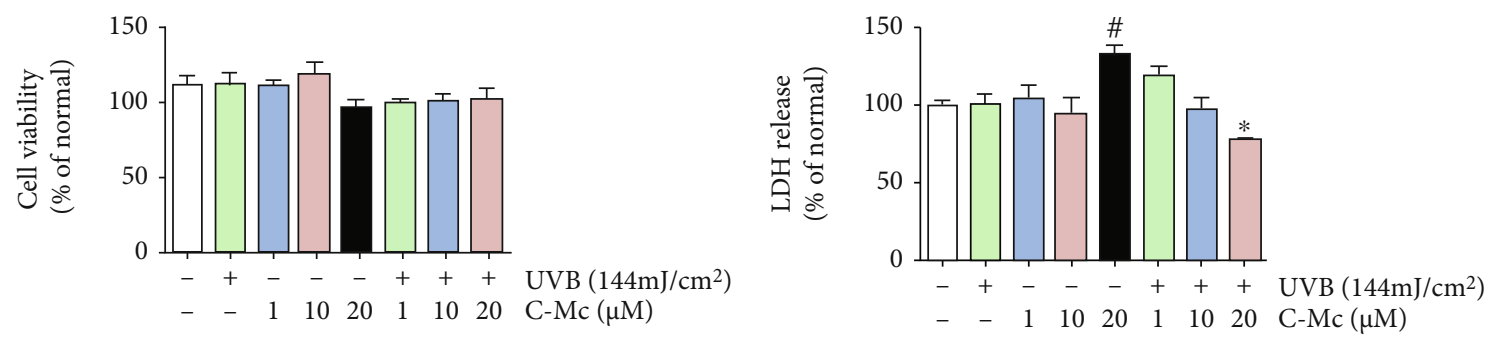

(e)

(f)

Figure 4: Continued. 


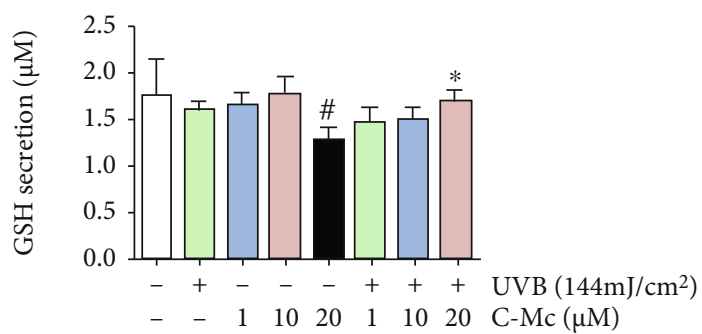

(g)

FIGURE 4: Free radical scavenging ability and cell viability of ginsenoside C-Mc. (a) Compound structure and physicochemical properties of ginsenoside C-Mc. The proper range of the chemical physicochemical properties is given by ADMETlab 2.0 [34]. (b-d) ROS production. (e) Cell viability. (f) LDH release. (g) GSH secretion. NHDFs were irradiated or nonirradiated with $144 \mathrm{~mJ} / \mathrm{cm}^{2} \mathrm{UVB}$, followed by treatment with the indicated of ginsenoside C-Mc $(1,10$, and $20 \mu \mathrm{M})$. All data are shown as the mean \pm SD of three independent experiments. \# and $*$ indicate significant differences from the nonirradiated control and UVB-irradiated control groups. ${ }^{\#} p<0.05,{ }^{\# \#} p<0.01$, and ${ }^{\# \# \#} p<0.001$ contrast with the nonirradiated control. ${ }^{*} p<0.05,{ }^{* *} p<0.01$, and ${ }^{* * *} p<0.001$ contrast with the UVB-irradiated control. ${ }^{*} p$ $<0.05,{ }^{* *} p<0.01$, and ${ }^{* * *} p<0.001$

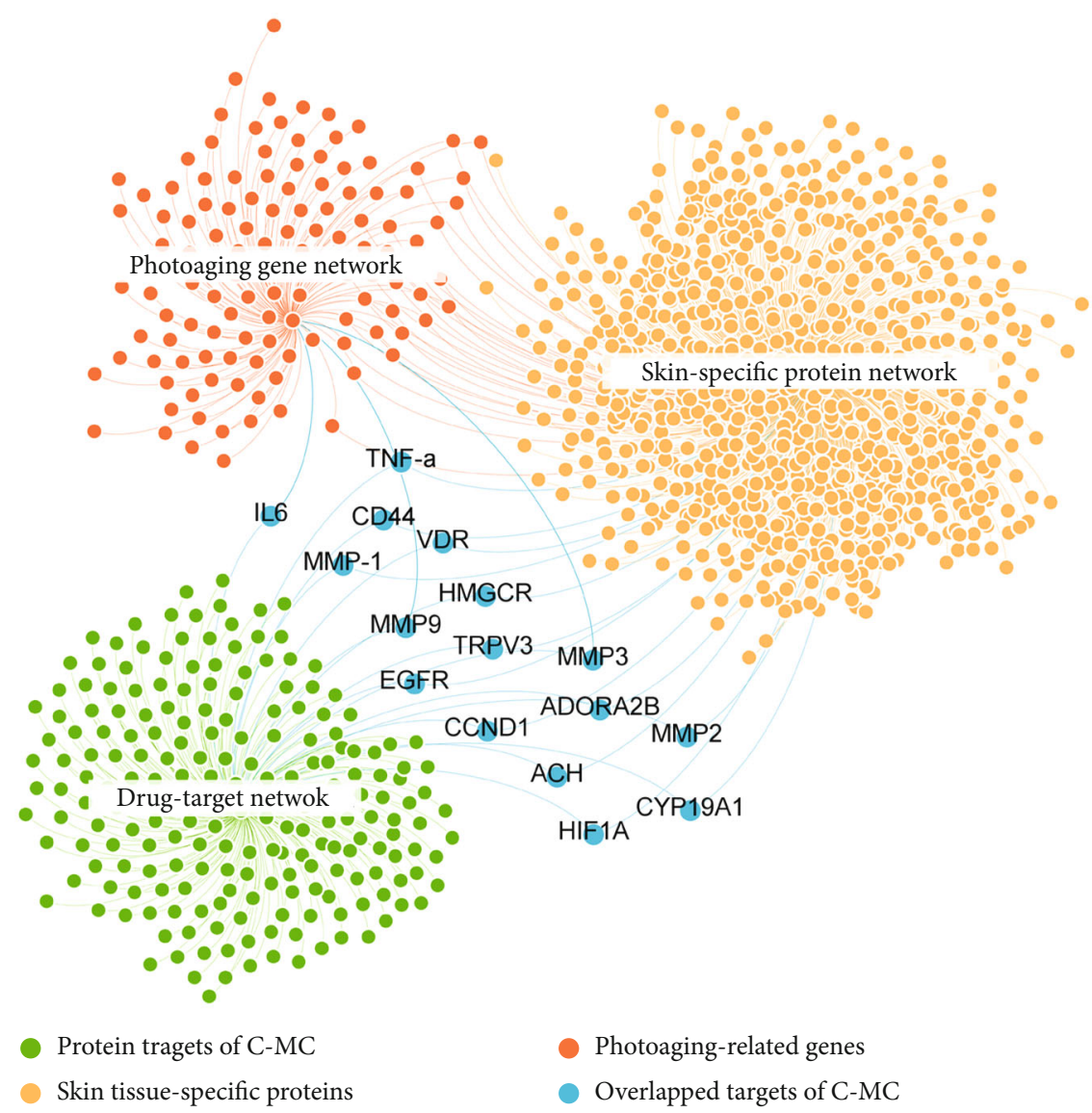

FIGURE 5: Global drug-target network of ginsenoside C-Mc interacted with photoaging-related gene network and skin tissue-specific expression protein network. Gene symbol names of the overlapped targets of C-Mc are displayed. The detailed information of the networks is provided in Supplementary Table S3-5.

were performed using a one-way analysis of variance (ANOVA) and Student's $t$-tests. Statistical significance was set at $p<0.05$.

\section{Results}

3.1. Preliminary Identification of Potential Antiphotoaging Ginsenosides Based on Shortest Distance Calculation in
PCA Space. On the basis of the 16 known antiphotoaging ginsenosides, we firstly figure out the rational boundary constraint of the space of PCA (Figure 2(a)). The maximum and minimum values of PCA 1, PCA 2, and PCA 3 of the 16 ginsenosides, respectively, were set as the thresholds for eliminating the improper compounds. As shown in Figure 2(b), 15 of the 82 candidate ginsenosides were removed in this 


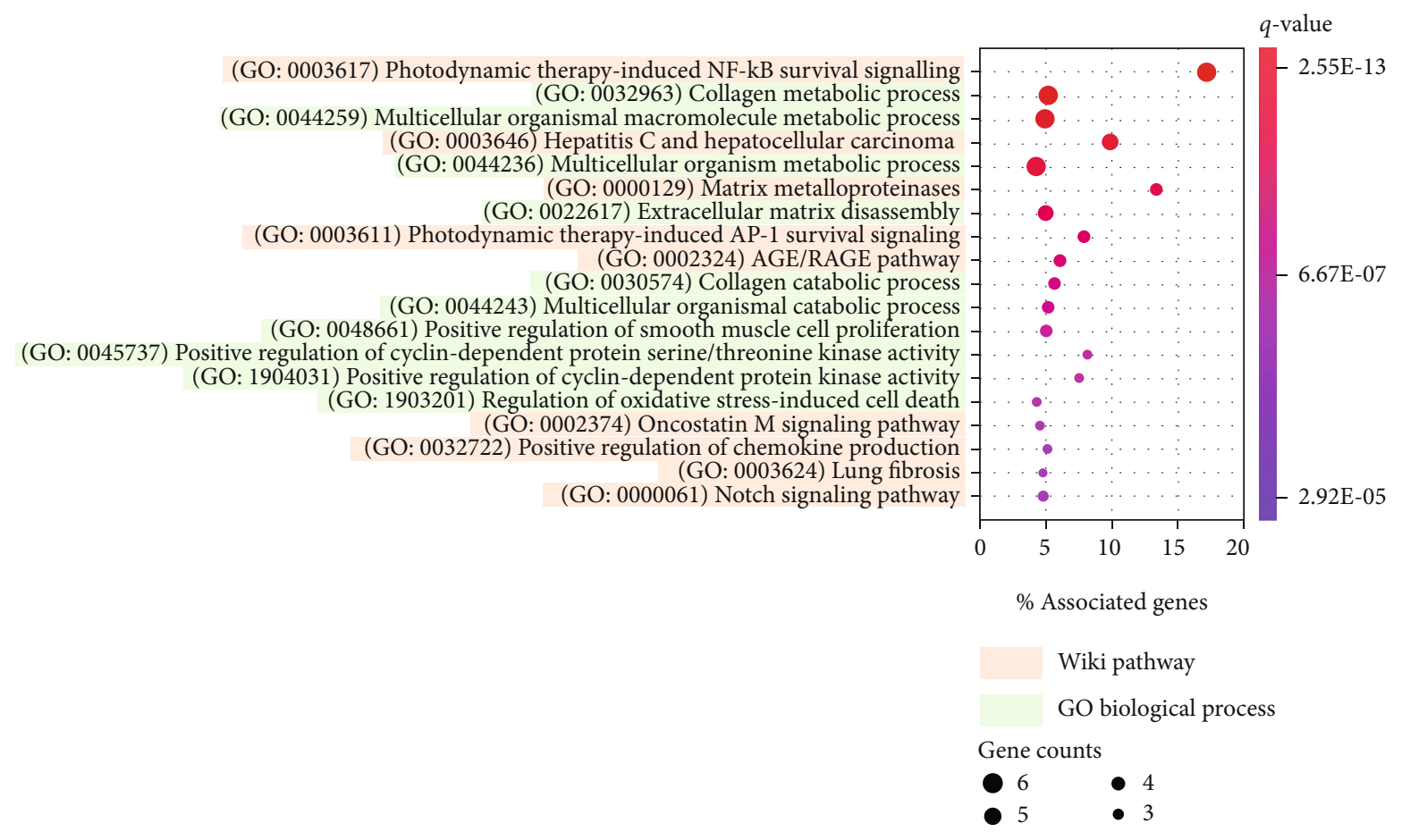

(a)

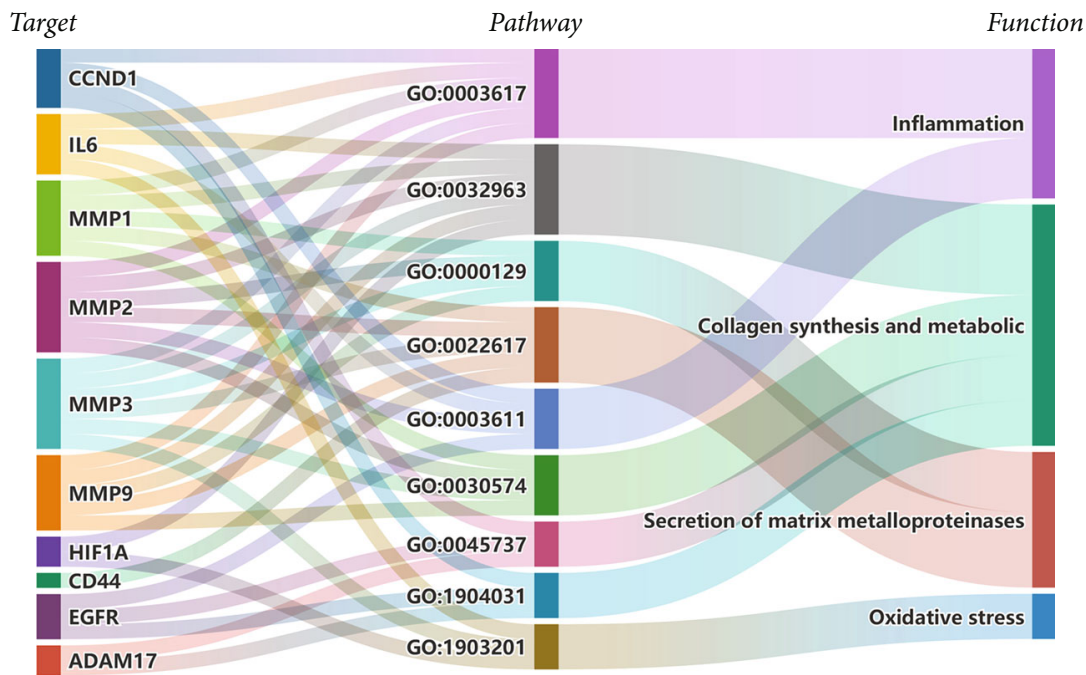

(b)

Figure 6: Gene enrichment analysis of the potential targets of ginsenoside C-Mc overlapped with photoaging and skin-specific genes. (a) Gene Ontology (GO) biological process enrichment and WikiPathway annotation; (b) Sankey diagram illustrating the relationship among the photoaging-relevant enriched pathways/processes, corresponding proteins, and biological function of C-Mc against photoaging.

step. After that, the 16 known antiphotoaging ginsenosides were fitted as a curve by a polynomial of third order in the PCA coordinate frame, and the shortest distance between each point of unknown ginsenoside and the fitted curve was calculated (Figure 2(c)). We identified 22 ginsenosides have a significant shortest distance $\left(D_{\min }<0.2\right)$ to the set of known active ginsenosides, which are preliminary identified as potential candidates for further study (Supplementary Table S7).

3.2. Candidates Prioritizing via Pairwise Chemical Structure Similarity Analysis. Compared to the above PCA based on molecular properties, the chemical similarity analysis conducting by molecular fingerprint can further screen out the more promising antiphotoaging ginsenosides from the perspective of compound structures. In this study, the similarity between the 16 known active ginsenosides and the remaining 22 unknown ginsenosides was measured individually. The average similarity index was calculated for each unknown ginsenosides by averaging the similarity results of 16 pairs of compounds. As presented in Figure 3(a), there are 10 ginsenosides possessing an average similarity index higher than 0.7, including ginsenosides C-Mc, XVII, CMx1, F2, Rs2, LXXV, Mx, Rs1, (20R)-ginsenoside Rh2, and 


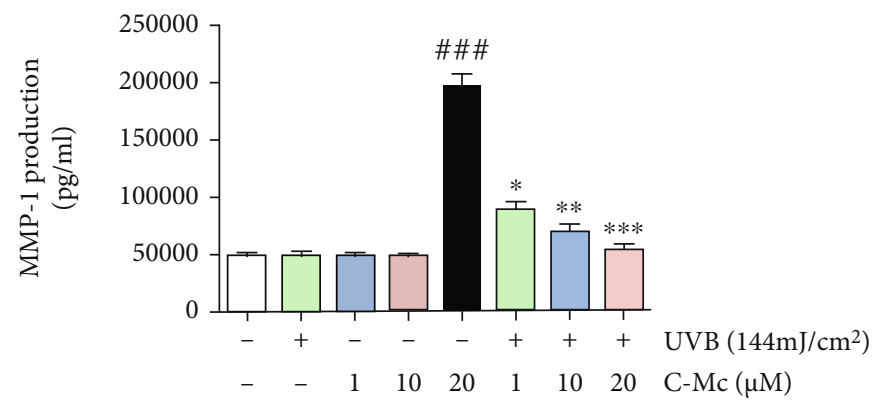

(a)

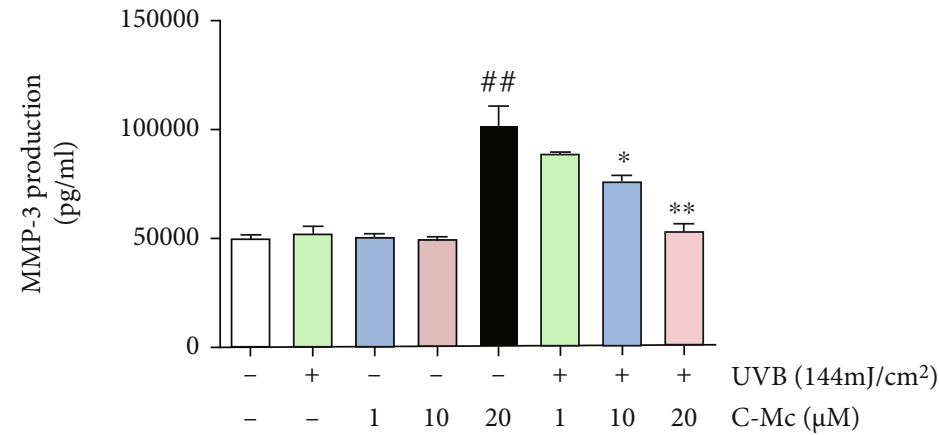

(b)

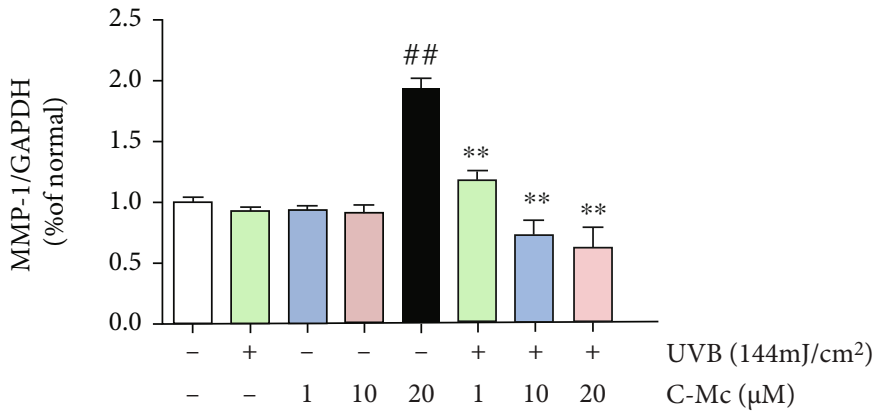

(c)

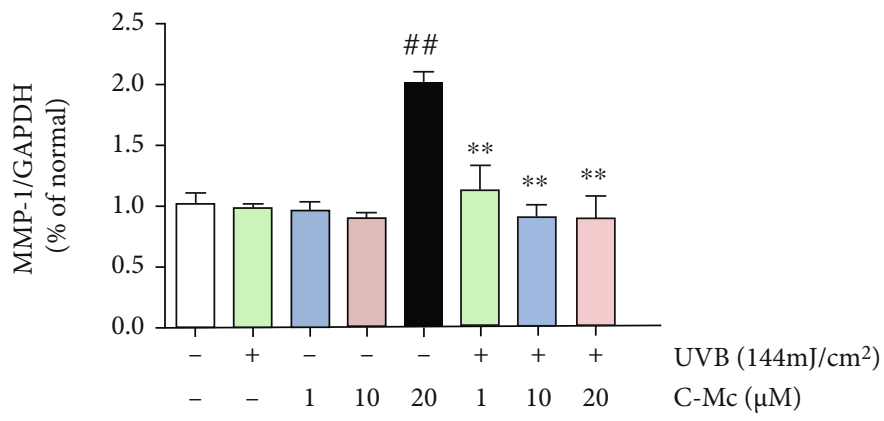

(d)

FIgUre 7: Continued. 


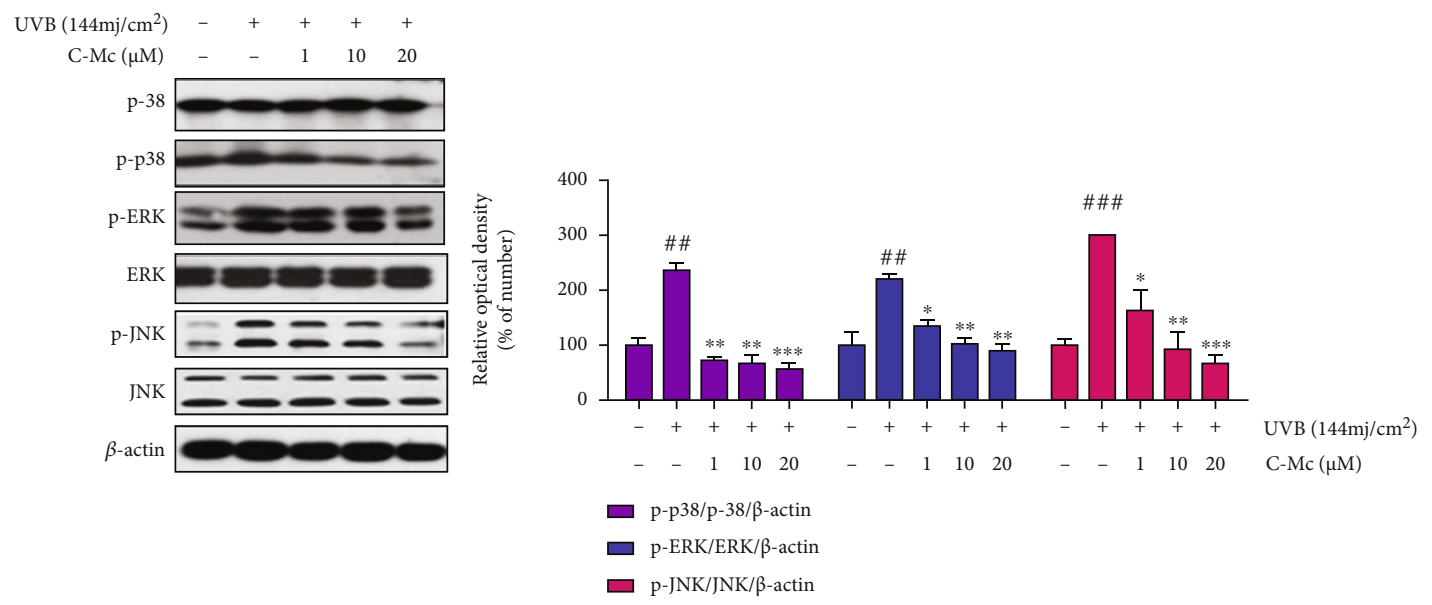

(e)
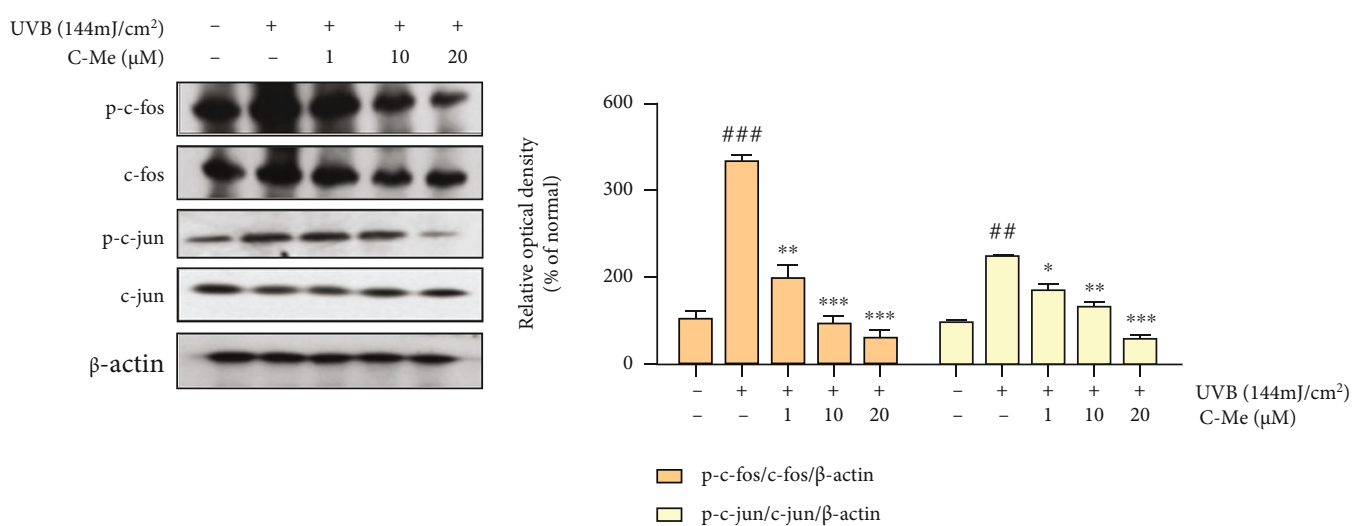

(f)

FIgure 7: C-Mc prevented secretion of MMP-1 and MMP-3 and inhibited MAPK/AP-1 signaling pathway in UVB-irradiated NHDFs. Production of (a) MMP-1 and (b) MMP-3 under non-UVB irradiation and UVB-irradiated conditions; (c) MMP-1 and (d) MMP-3 mRNA expression. An equimolar quantity of mRNA was quantified compared to GAPDH. Cells were incubated in absence or presence of ginsenoside C-Mc at the present concentration after exposure to UVB radiation $\left(144 \mathrm{~mJ} / \mathrm{cm}^{2}\right)$. (e) The protein levels of p38, ERK, and JNK in UVB-irradiated NHDFs measured by Western blot analysis. NHDFs were irradiated or nonirradiated with UVB, followed by treated with ginsenoside C-Mc for $1 \mathrm{~h}$ (MAPK). The signal intensities for phosphorylation levels of p38, ERK, and JNK. (f) The protein levels of c-Fos and c-Jun in UVB-irradiated NHDFs measured by Western blot analysis. NHDFs were irradiated or nonirradiated with UVB, followed by treatment with C-Mc for $4 \mathrm{~h}$ (AP-1). The signal intensities for phosphorylation levels of c-Fos and c-Jun. Values shown are the mean $\pm \mathrm{SD}$. \# and $*$ indicate significant differences from the nonirradiated control and UVB-irradiated control groups. ${ }^{\#} p<0.05,{ }^{\# \#} p<0.01$, and ${ }^{\# \#} p<0.001$ contrast with the nonirradiated control. ${ }^{*} p<0.05,{ }^{* *} p<0.01$, and ${ }^{* * *} p<0.001$ contrast with the UVB-irradiated control.

NSC308876, which are supposed to have higher possibilities as photoprotective agents. The structures of the 10 ginsenosides are provided in Figure 3(b).

\subsection{Drug-Likeness Evaluation and ADMET Screening for the} Candidates. It is widely recognized that drug-likeness evaluation and ADMET screening are the essential steps in the early stage of drug development that help to avoid latestage failures $[43,44]$. Thus, before the wet-lab experiments, a comprehensive in silico evaluation was conducted on the 10 predicted antiphotoaging ginsenosides. As listed in Table 1, all of them were predicted to be positive by the drug-likeness model and had passed the PAINS frequent hitter filters. They also showed low toxicity according to the results of AOT and skin sensitization prediction. For PPB, one of the major indicators reflecting the ability of drug uptake and distribution, these candidates except (20R)-ginsenoside Rh2 were considered to have a proper property with predicted value $<90 \%$. However, in the evaluation of human oral bioavailability $\left(F_{20 \%}\right)$, six of the ten ginsenosides were labeled as poor that may have low efficiency of the drug delivery to the systemic circulation. Put together, there are three ginsenosides (C-Mc, F2, and $\mathrm{Mx}$ ) have passed all the evaluation of drug-likeness and ADMET properties, which could be served as the most promising antiphotoaging drug candidates of ginsenosides.

3.4. In Vitro Validation of the Cytoprotective Activity of Ginsenoside $C-M c$ against UVB-Irradiated Photodamage. As the in silico prediction suggested, ginsenosides C-Mc, $\mathrm{F} 2$, and $\mathrm{Mx}$ possess high potential as novel antiphotoaging agents. Comprehensively considering the extractability and 


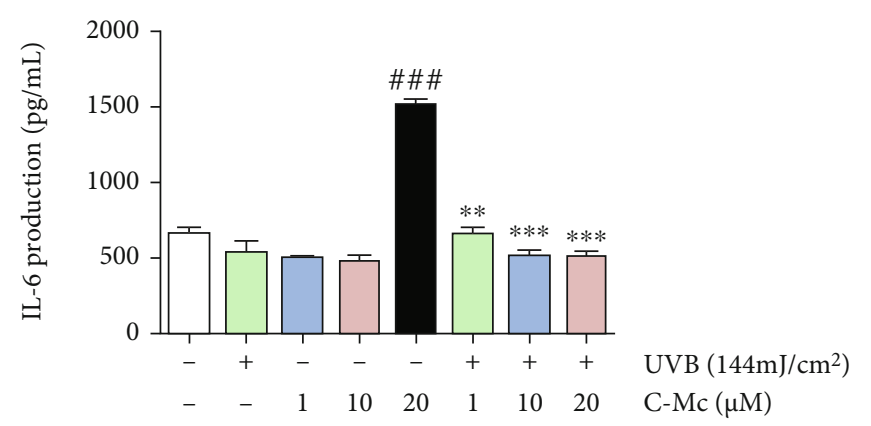

(a)

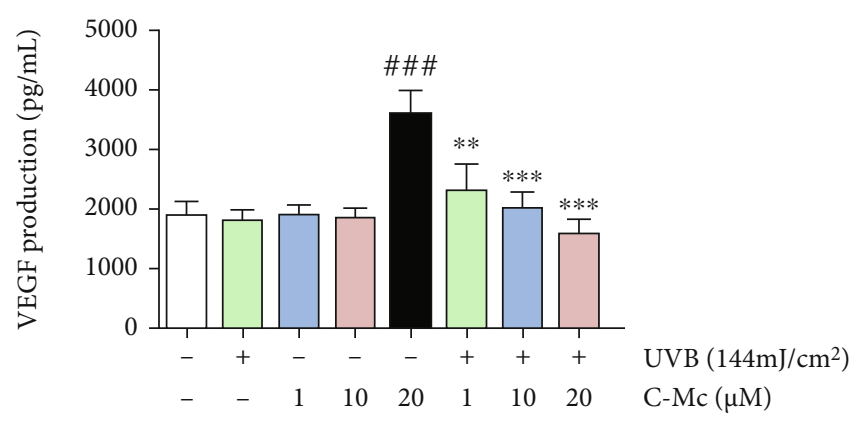

(b)

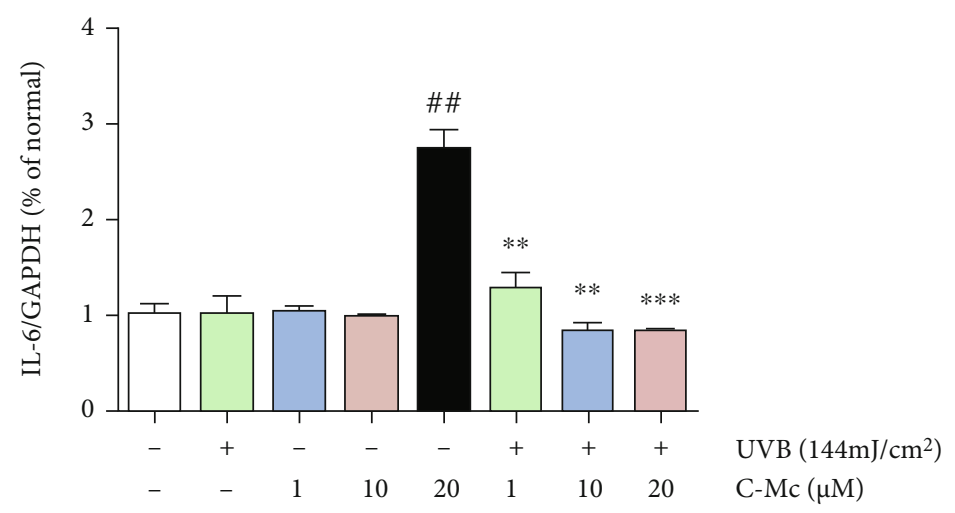

(c)
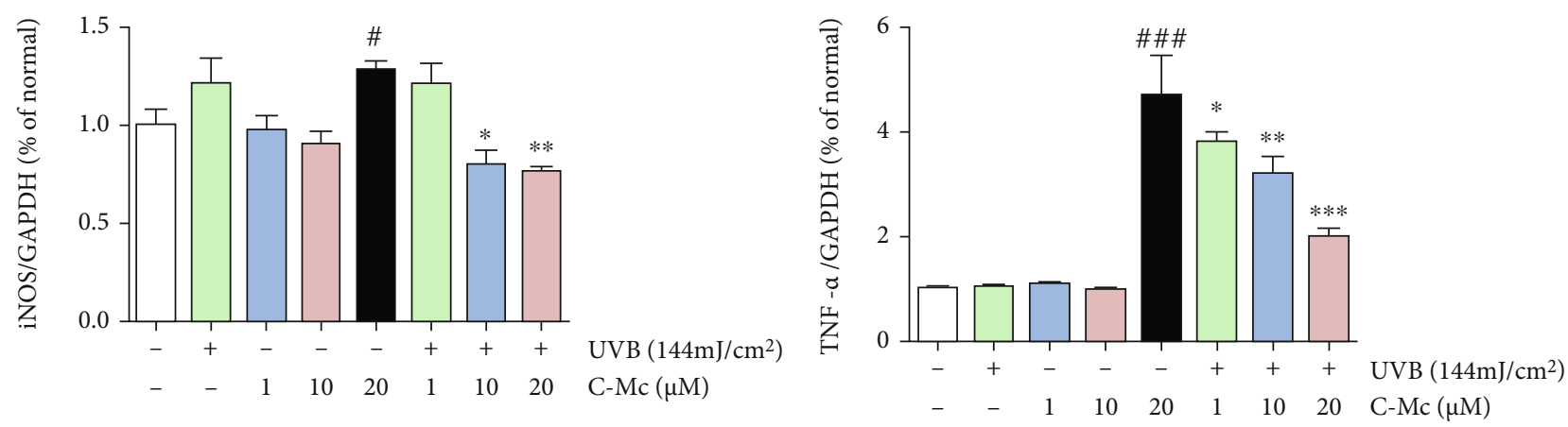

(d)

(e)

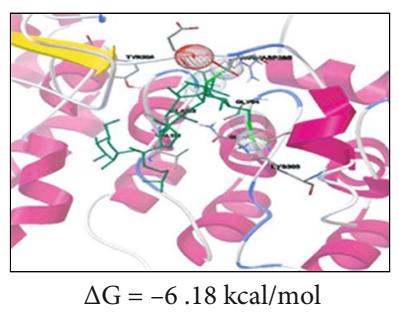

(f)

FIgure 8: Continued. 


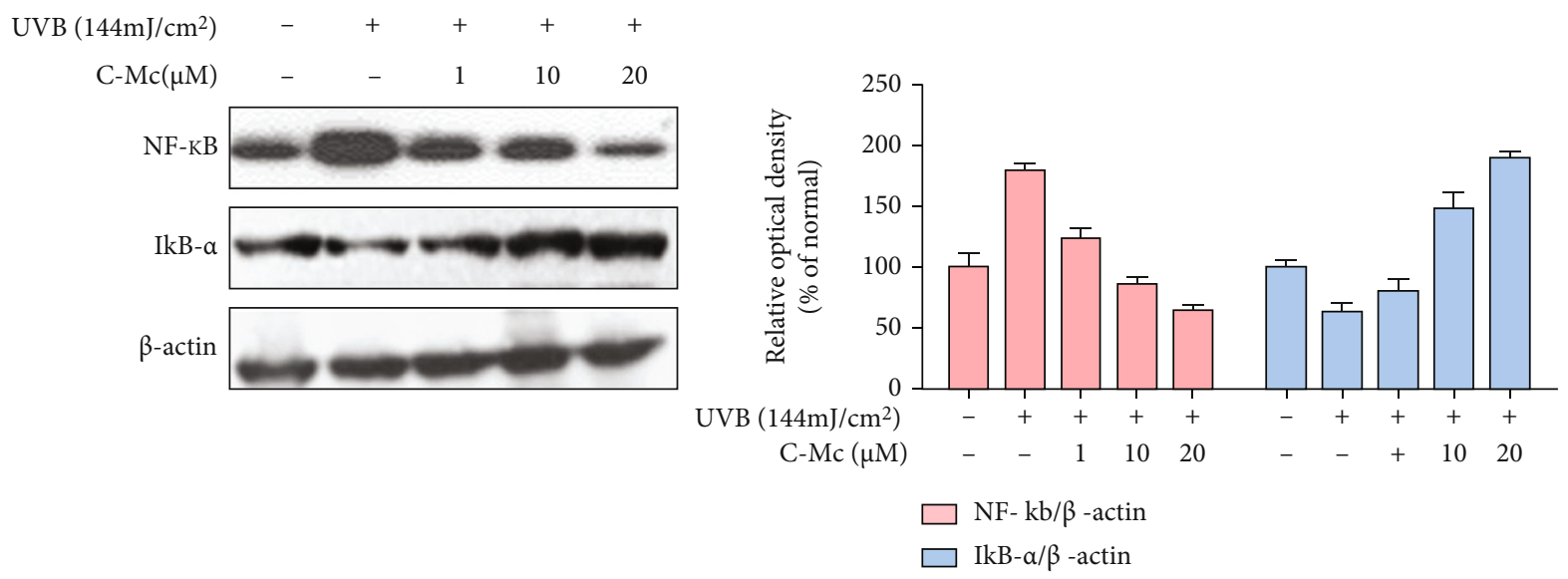

(g)

Figure 8: Ginsenoside C-Mc inhibited UVB-induced inflammatory cytokine secretion and alleviated UVB-induced NF- $\kappa \mathrm{B} / \mathrm{I} \kappa \mathrm{B}-\alpha$ expression. Production of (a) IL-6 and (b) VEGF under non-UVB irradiation and UVB-irradiated conditions; (c) IL-6, (d) iNOS, and (e) TNF- $\alpha$ mRNA expression. An equimolar quantity of mRNA was quantified compared to GAPDH. Cells were incubated in absence or presence of ginsenoside C-Mc at the present concentration after exposure to UVB radiation $\left(144 \mathrm{~mJ} / \mathrm{cm}^{2}\right.$ ). (f) Molecular docking simulation for C-Mc binding to NF- $\kappa$ B. (g) The protein levels of NF- $\kappa$ B and $\mathrm{I} \kappa \mathrm{B}-\alpha$ detected by Western blot. Values shown are the mean $\pm \mathrm{SD}$. \# and $*$ indicate significant differences from the nonirradiated control and UVB-irradiated control groups. ${ }^{\#} p<0.05,{ }^{\# \#} p<0.01$, and ${ }^{\# \# \#} p<0.001$ contrast with the nonirradiated control. ${ }^{*} p<0.05,{ }^{* *} p<0.01$, and ${ }^{* * *} p<0.001$ contrast with the UVB-irradiated control.

compound properties, we finally selected ginsenoside C-Mc to validate its photoprotective activity in NHDFs (Figure 4(a)).

Confirmation of ginsenoside $C-M c$. The purity of ginsenoside C-Mc was over $95.02 \%$ as detected by HPLC (Supplementary Figure S3a). C-Mc yielded a $[\mathrm{M}+\mathrm{Na}]^{+}$at $m / z 777$ or $[\mathrm{M}+\mathrm{H}]^{+}$at $m / z 755$ and $\mathrm{MS}^{2}$ ions at $605\left[\mathrm{M}+\mathrm{H}-\mathrm{Araf}-\mathrm{H}_{2} \mathrm{O}\right]^{+}$, $443\left[\mathrm{M}+\mathrm{H}-\text { Araf-Glc- } \mathrm{H}_{2} \mathrm{O}\right]^{+}$, and $425[\mathrm{M}+\mathrm{H}$-Araf-Glc$\left.2 \mathrm{H}_{2} \mathrm{O}\right]^{+}$(Supplementary Figure S3b) $[45,46]$.

Antioxidant ability of $C-M c$. In general, UVB exposure increases ROS generation in cells. This phenomenon was reversed after treatment with $\mathrm{C}-\mathrm{Mc}$; at a concentration of $20 \mu \mathrm{M} \mathrm{C}-\mathrm{Mc}$, the ROS levels were reduced by $88.96 \%$ (Figures 4(b)-4(d)).

Toxicity of ginsenoside $C-M c$ in NHDFs. An MTT assay was performed to test NHDF viability. As presented in Figures 4(e), C-Mc showed no toxicity with concentrations of 1,10 , and $20 \mu \mathrm{M}$ in nonirradiated and irradiated NHDFs. Interestingly, C-Mc treatment at concentrations as high as $20 \mu \mathrm{M}$ unexpectedly increased cell viability in the nonirradiated groups. The broader concentration range $(1,10,20,30$, 50,100 , and $200 \mu \mathrm{M}$ ) for toxicity measurement is provided in Supplementary Figure S4.

Cytoprotective activity of $\mathrm{C}-\mathrm{Mc}$ in UVB-irradiated NHDFs. As shown in Figure 4(f), UVB-exposed groups showed significantly enhanced $\mathrm{LDH}$ release, whereas treatment with ginsenoside $\mathrm{C}-\mathrm{Mc}$ reduced the release of $\mathrm{LDH}$. Because of its antioxidant properties, glutathione (GSH) is an essential factor in maintaining cellular redox homeostasis and preventing UV-induced oxidative stress damage [47]. As expected, after UVB irradiation, the intracellular GSH was notably exhausted, while C-Mc $(1,10$, and $20 \mu \mathrm{M})$ treatment reversed this trend. In particular, $20 \mu \mathrm{M}$ C-Mc enabled recovery of GSH level to almost the control value (Figure $4(\mathrm{~g})$ ).
3.5. System Pharmacology Analysis for Exploration of the Molecular Mechanisms of C-Mc against Photoaging. There are currently no scientific data on the detailed molecular mechanism of the rare ginsenoside $\mathrm{C}-\mathrm{Mc}$ in UVBinduced photoaging. Here, we performed system pharmacology analysis to explore the molecular mechanisms of $\mathrm{C}-\mathrm{Mc}$ against photoaging through integrating drug-target network, photoaging-related gene network, and skin tissue-specific expression protein network. As shown in Figure 5, the D-T network contains 222 DTIs, of which 3 targets of C-Mc are interacted with photoaging-related protein network and 13 are connected to skin-specific protein network. The 16 targets overlapped with photoagingrelated and skin-specific genes suggest the potential regulatory protein network of $\mathrm{C}-\mathrm{Mc}$ against photoaging. We thus further conducted gene enrichment analysis on these overlapped targets using ClueGO plug-in of Cytoscape (v3.2.0) [48]. As presented in Figure 6(a), the gene enrichment analysis annotated 9 pathways from WikiPathways and 11 biological processes from Gene Ontology (GO) with corrected $p$ values less than 0.01 ( $q$, corrected with Bonferroni step down). We found that most of the enriched pathways/processes have high correlation with photodamage. For instance, it is reported that the secretion of matrix metalloproteinases (MMPs) associated with degradation of extracellular matrix (ECM) proteins promotes UVB radiation-irradiated photodamage [49], which is consistent with the predicted pathways such as GO:0032963 (collagen metabolic process, $q=4.35 E-10$ ) and GO:0000129 (matrix metalloproteinases, $q=2.72 E-$ 08). Besides, as annotated by WikiPathway, these potential targets of C-Mc are involved in photodynamic therapyinduced AP-1 (GO:0003611, $q=1.97 E-07)$ and NF- $\kappa \mathrm{B}$ (GO:0003617, $q=2.55 E-13$ ) survival signaling pathway. Previous studies demonstrated that $\mathrm{AP}-1$ and NF- $\kappa \mathrm{B}$ are 


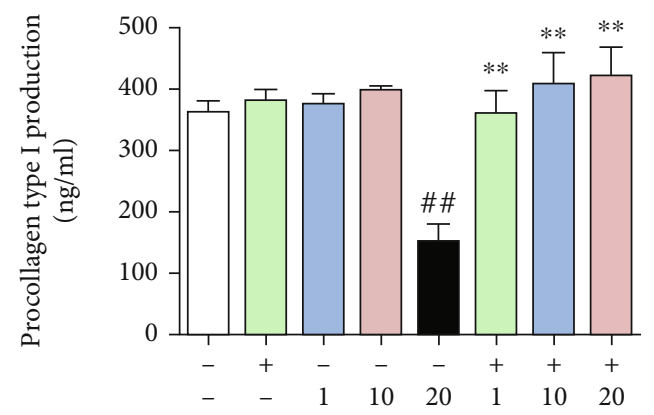

(a)

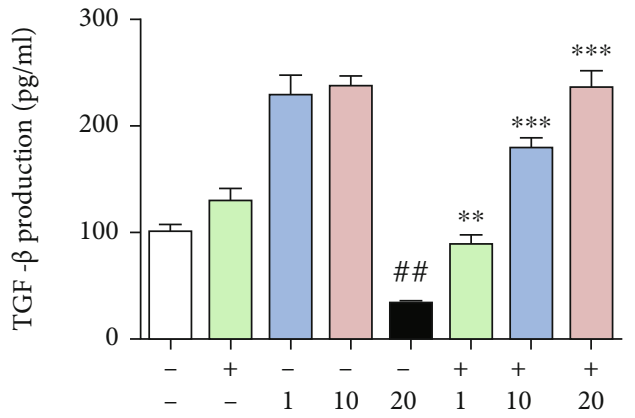

(b)

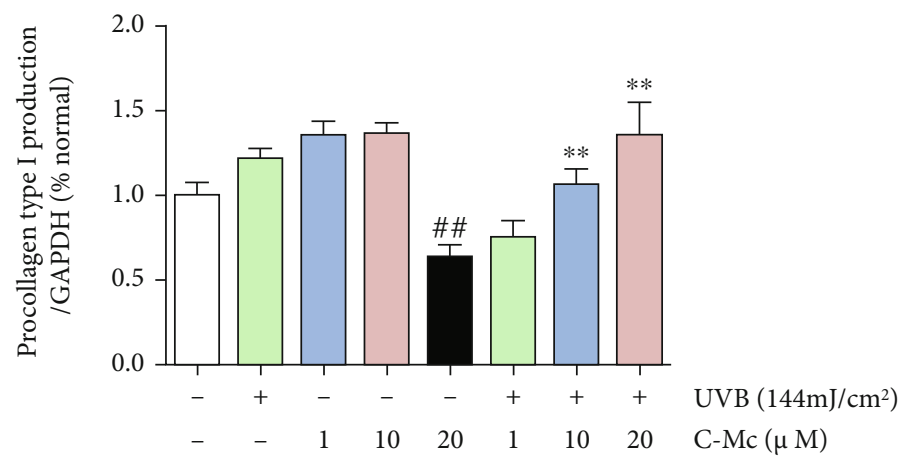

(c)

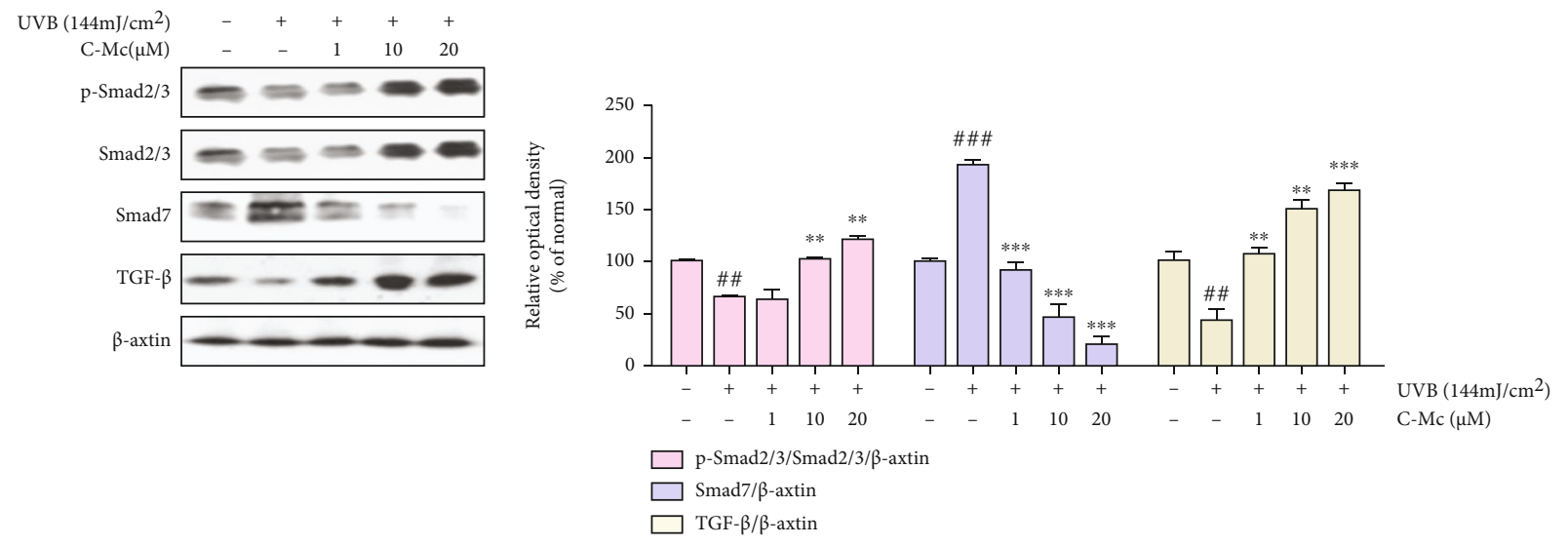

(d)

FIGURE 9: C-Mc increased in TGF- $\beta 1$ and procollagen type I secretion and regulated TGF- $\beta /$ Smad pathway in UVB-irradiated NHDFs. Production of (a) procollagen type I and (b) TGF- $\beta 1$ under non-UVB irradiation and UVB-irradiated conditions. (c) Procollagen type I mRNA expression. An equimolar quantity of mRNA was quantified compared to GAPDH. Cells were incubated in absence or presence of ginsenoside C-Mc at the present concentration after exposure to UVB radiation $\left(144 \mathrm{~mJ} / \mathrm{cm}^{2}\right)$. (d) The protein levels of Smad2/3, Smad7, and TGF- $\beta 1$ in UVB-irradiated NHDFs were measured by Western blot analysis. The NHDFs were irradiated or nonirradiated with UVB, followed by treated with ginsenoside C-Mc for $1.5 \mathrm{~h}$. The signal intensities for phosphorylation levels of Smad2/3, Smad7, and TGF- $\beta 1$ were tested by Western blotting. Values shown are the mean \pm SD. \# and $*$ indicate significant differences from the nonirradiated control and UVB-irradiated control groups. ${ }^{\#} p<0.05,{ }^{\# \#} p<0.01$, and ${ }^{\# \# \#} p<0.001$ contrast with the nonirradiated control. ${ }^{*} p<0.05,{ }^{* *} p<0.01$, and ${ }^{* * *} p<0.001$ contrast with the UVB-irradiated control.

essential for MMP-1 upregulation and ECM degradation $[50,51]$.

We further classified the photoaging-relevant enriched pathways/processes and corresponding proteins to elucidate the major biological function of C-Mc against photoaging (Figure 6(b)). Obviously, these pathways share a lot of common proteins such as MMPs (MMP-1, MMP-2, MMP-3, and MMP-9), indicating that C-Mc may exert multitarget synergistic antiphotoaging effect via acting on these potential key targets. The biological pathways and processes can be summarized into four important functional modules related to photoaging, including inflammation, collagen synthesis and metabolic, secretion of MMPs, and oxidative stress (Figure 6(b)). In fact, the four functional modules are intrinsically connected. UVB radiation induces secretion of MMPs and further leads to the loss of collagen in the 

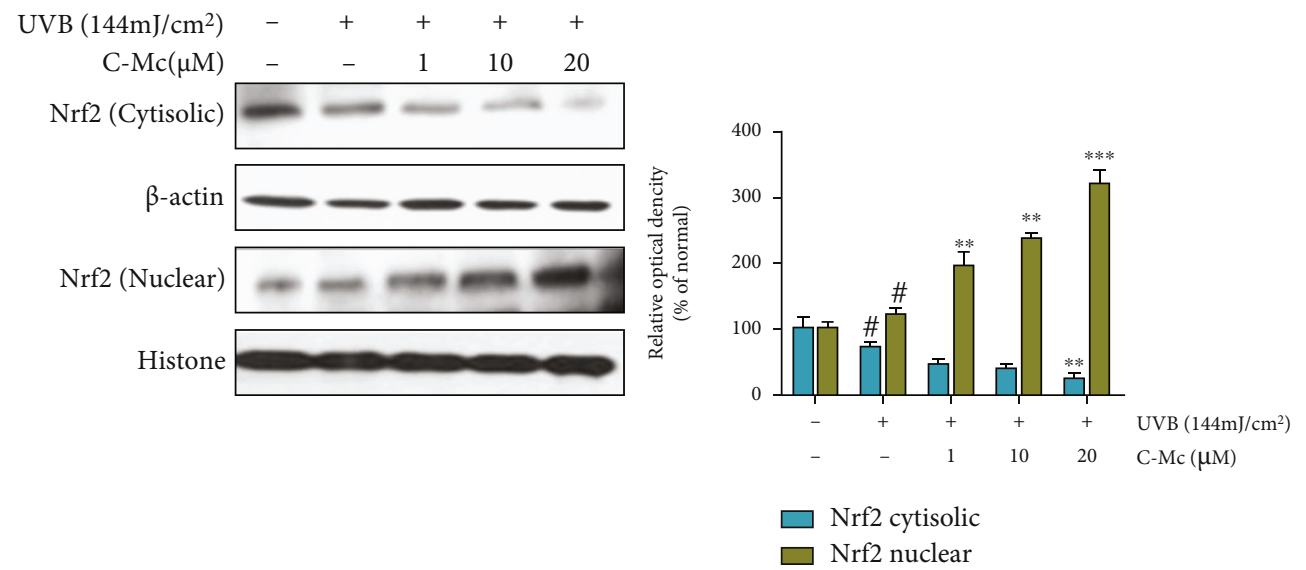

(a)

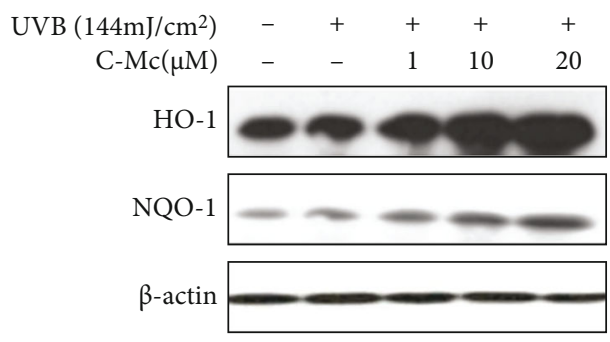

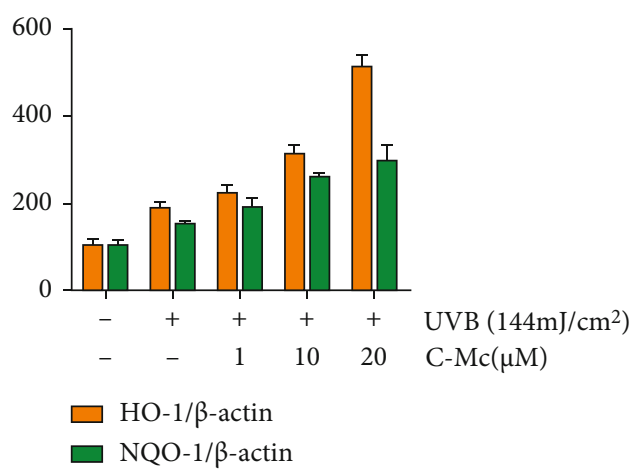

(b)

Figure 10: Effects of ginsenoside C-Mc on Nrf2, HO-1, and NQO-1 expression in UVB-irradiated NHDFs. (a) The protein levels of Nrf2 in UVB-irradiated NHDFs were measured by Western blot analysis. The signal intensities for phosphorylation levels of Nrf2. The protein levels of Nrf2 in UVB-irradiated NHDFs were measured by Western blot analysis. (b) The signal intensities for phosphorylation levels of HO-1 and NQO-1. The NHDFs were irradiated or nonirradiated with UVB, followed by treated with ginsenoside C-Mc for $3 \mathrm{~h}$. The results were shown as the mean $\pm \mathrm{SD}$ of at least three independent experiments. ${ }^{\#} p<0.05,{ }^{\# \#} p<0.01$, and ${ }^{\# \#} p<0.001$ contrast with the nonirradiated control. ${ }^{*} p<0.05,{ }^{* *} p<0.01$, and ${ }^{* * *} p<0.001$ contrast with the UVB-irradiated control.

dermal ECM via activation of the mitogen-activated protein kinases (MAPK)/activator protein-1 (AP-1) pathway [49]. AP-1 is responsible for activating the expression of MMPs, which cause degradation of ECM-related proteins, and synthesis of collagen and elastin [50]. Several studies have demonstrated that the TGF- $\beta /$ Smad pathway regulates procollagen synthesis [52]. Furthermore, UV radiation stimulates NF- $\kappa \mathrm{B}$ proteins through suppression of the synthesis of $\mathrm{I} \kappa \mathrm{B}-\alpha$ [53]. Activated NF- $\kappa \mathrm{B}$ regulates the release of proinflammatory cytokines, which play a critical role in upregulation of MMP-1 and ECM degradation [51]. Moreover, in skin exposed to UVB, overexpression of proinflammatory cytokines such as TNF- $\alpha$, iNOS, and IL-6 further activates NF- $\kappa \mathrm{B}$ by enhancing its translocation to the nucleus, resulting in overexpression of vascular endothelial growth factor (VEGF) [54]. In view of previous findings, anti-inflammation, antioxidative, expediting collagen synthesis and prevention metabolic, and inhibiting the secretion of MMPs might be considered as the underlying mechanism of actions of $\mathrm{C}-\mathrm{Mc}$ to prevent photoaging damage.
3.6. Experimental Validation of the Antiphotoaging Mechanisms of Ginsenoside $C-M c$ in UVB-Irradiated NHDFs. As the system pharmacology analysis revealed ginsenoside $\mathrm{C}-\mathrm{Mc}$ might exert antiphotoaging effect by influencing four major functional modules, including inflammation, collagen synthesis and metabolic, secretion of MMPs, and oxidative stress (Figure 6). In the following sections, we further conducted experiments in UVBirradiated NHDFs under the guidance of system pharmacology predictions, to systematically elucidate the influence of $\mathrm{C}-\mathrm{Mc}$ on these functional modules and relevant signal pathways, aiming to better understand the underlying mechanism of actions of C-Mc against photoaging.

3.7. C-Mc Prevented Secretion of MMP-1 and MMP-3 and Inhibited MAPK/AP-1 Signaling Pathway in UVBIrradiated NHDFs. System pharmacology analysis indicated that the secretion of MMPs is one of the major functional modules modulated by ginsenoside C-Mc. As shown in Figures 7 (a) and 7(b), exposure of NHDFs to UVB irradiation $\left(144 \mathrm{~mJ} / \mathrm{cm}^{2}\right)$ promoted secretion of MMP-1 and 


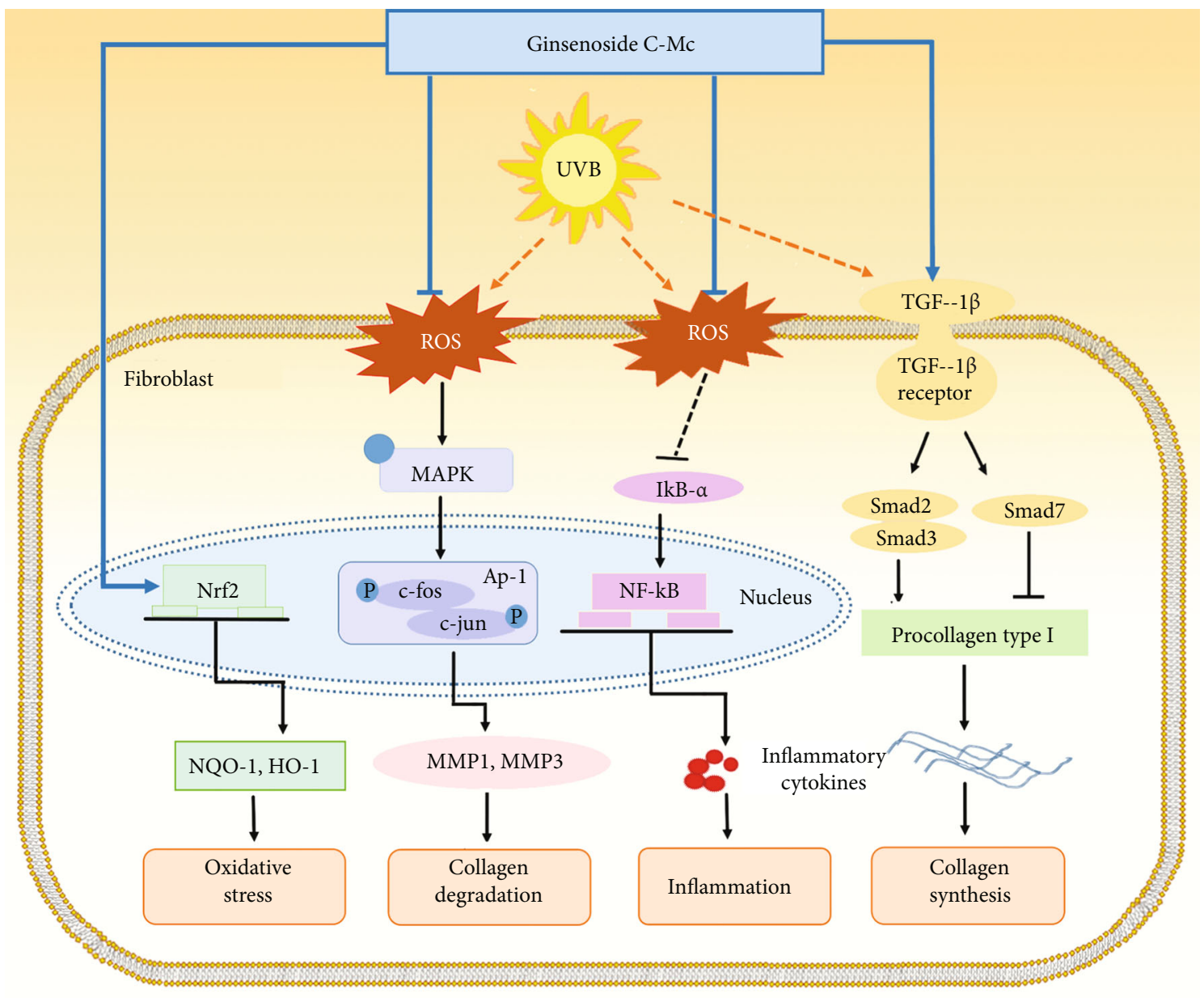

FIGURE 11: Schematic summary illustrating amelioration of ginsenoside C-Mc on photoaging in UVB-irradiated NHDFs via modulating oxidation stress, inflammation, matrix metalloproteinase secretion, collagen degradation, and synthesis.

MMP-3, whereas treatment with C-Mc from 1 to $20 \mu \mathrm{M}$ significantly blocked MMP-1 and MMP-3 production. C-Mc decreased MMP-1 and MMP-3 secretion by $70.53 \%$ and $56.39 \%$, respectively.

We evaluated MMP-1 and MMP-3 mRNA levels in UVB-exposed NHDFs by real-time PCR (Figures 7(c) and $7(d))$. Our results indicated that the increased expression of MMP-1 and MMP-3 mRNAs induced by UVB irradiation was decreased by treatment with C-Mc. Specifically, $20 \mu \mathrm{M}$ C-Mc suppressed UVB-induced MMP-1 and MMP-3 expression by $68.35 \%$ and $49.55 \%$, respectively. Moreover, $20 \mu \mathrm{M}$ C-Mc treatment upregulated procollagen type I expression by $198.36 \%$. Consistent with the q-PCR data, ELISA results showed that expression of MMP-1 and MMP-3 proteins was strongly increased after UVB exposure whereas $\mathrm{C}-\mathrm{Mc}$ reversed this trend and markedly downregulated the production of MMP-1 and MMP-3.

As predicted by the gene enrichment analysis, the MAPK/AP-1 signaling pathway might be regulated by CMc, which is closely relevant to the expression of MMPs. To better understand the molecular mechanisms of $\mathrm{C}-\mathrm{Mc}$, we examined the MAPK family in NHDFs. Cells treated with UVB radiation showed specific increases in the phosphorylation of p38, ERK, and JNK compared with nonirra- diated cells (Figure 7(e)). Treatment with C-Mc suppressed phosphorylation of these MAPKs in a dose-dependent manner. In particular, treatment with $20 \mu \mathrm{M}$ C-Mc reduced pp38, p-ERK, and p-JNK expression by $65.3 \%, 72.1 \%$, and $60.23 \%$ inhibition, respectively. We next evaluated the phosphorylation of AP-1 by Western blotting. As shown in Figure $7(\mathrm{f})$, UVB radiation triggered phosphorylation of both c-Jun and c-Fos proteins. Compared with nonirradiated cells, the p-c-Jun and p-c-Fos levels were $167.15 \%$ and $175.77 \%$ higher, respectively, in UVB-exposed cells whereas treatment with C-Mc $(20 \mu \mathrm{M})$ noticeably reversed this effect to levels of $104 \%$ and $83 \%$, respectively.

3.8. C-Mc Inhibited UVB-Induced Inflammatory Cytokine Secretion and Alleviated $N F-\kappa B / I \kappa B-\alpha$ Expression. To validate the effects of C-Mc on inflammation functional module, we tested the changes of inflammatory cytokine secretion in UVB-irradiated NHDFs. As presented in Figures 8(a) and 8(b), exposure of NHDFs to UVB irradiation $\left(144 \mathrm{~mJ} / \mathrm{cm}^{2}\right)$ promoted secretion of IL-6 and VEGF, whereas treatment with C-Mc from 1 to $20 \mu \mathrm{M}$ significantly blocked IL-6 and VEGF production. Ginsenoside C-Mc quenched decreased VEGF secretion by $52.7 \%$. 
We performed q-PCR to assess the effects of C-Mc on iNOS, TNF- $\alpha$, and IL-6 expression in NHDFs. To quantify the results, the ratio of iNOS/GAPDH, TNF- $\alpha / G A P D H$, and IL-6/GAPDH in non-UVB irradiated cells was set to 1.0, according to the signal intensity. In the UVB-exposed group, the mRNA levels of iNOS, TNF- $\alpha$, and IL- 6 obviously increased as expected, and this trend was dramatically reversed after C-Mc treatment (Figures 8(c)-8(e)). After treatment with $20 \mu \mathrm{M}$ C-Mc, expression of the proinflammatory cytokines iNOS, IL-6, and TNF- $\alpha$ was downregulated by $61.22 \%, 60.97 \%$, and $59.88 \%$, respectively.

System pharmacology analysis indicated the important role of $\mathrm{C}-\mathrm{Mc}$ in the modulation of $\mathrm{NF}-\kappa \mathrm{B} / \mathrm{I} \kappa \mathrm{B}-\alpha$ pathway. Besides, molecular docking simulation also showed that $\mathrm{C}$ $\mathrm{Mc}$ has high affinity to $\mathrm{NF}-\kappa \mathrm{B}$ with relatively low binding energy $(\Delta G)$ of $-6.18 \mathrm{kcal} / \mathrm{mol}$, indicating that it may directly involve in the regulation of related proteins (Figure 8(f) and Supplementary Table S2). To verify these hypotheses, we evaluated the expression of NF- $\kappa \mathrm{B}$ and $\mathrm{I} \kappa \mathrm{B}-\alpha$ by Western blot analysis. Compared with the UVB-exposed control, ginsenoside $\mathrm{C}-\mathrm{Mc}$ inhibited NF- $\kappa \mathrm{B}$ expression in a dosedependent manner and markedly increased $\mathrm{I} \kappa \mathrm{B}-\alpha$ protein expression (Figure 8(g)).

3.9. C-Mc Increased in TGF- $\beta 1$ and Procollagen Type I Secretion and Regulated TGF- $\beta /$ Smad Pathway in UVBIrradiated NHDFs. In addition to the inhibition of MMPs and inflammatory cytokine secretion, expediting collagen synthesis is another important functional module of C-Mc predicted by system pharmacology analysis. As shown in Figures $9(\mathrm{a})-9(\mathrm{c})$, we found that C-Mc treatment affected secretion of TGF- $\beta 1$ and type I procollagen; the levels of type I procollagen and TGF- $\beta 1$ increased by $347 \%$ and $294 \%$, respectively, after C-Mc treatment.

The TGF- $\beta /$ Smad signaling pathway plays a crucial role in promoting procollagen synthesis. We examined this pathway in NHDFs after exposure to UVB $\left(144 \mathrm{~mJ} / \mathrm{cm}^{2}\right)$ and treatment with different concentrations of C-Mc for $1.5 \mathrm{~h}$. As expected, the levels of phosphorylated $\mathrm{Smad} 2 / 3$ and TGF- $\beta 1$ (Figure $9(\mathrm{~d})$ ) were drastically upregulated in cells treated with ginsenoside $\mathrm{C}-\mathrm{Mc}$, consistent with previous findings.

3.10. Effect of C-Mc on the Nrf2/ARE Signaling Pathway Relevant to Oxidative Stress. As the above biological process enrichment and antioxidation experiment results shown, CMc possesses significant antioxidant ability against UVBinduced ROS production. To elucidate the detailed mechanism of $\mathrm{C}-\mathrm{Mc}$, we further validate the action of $\mathrm{C}-\mathrm{Mc}$ towards the Nrf2/ARE signaling pathway. Nrf2/ARE signaling is the major regulatory pathway responsible for oxidative stress and further suppresses activation of the NF- $\kappa$ B pathway [55]. We evaluated the level of cytosolic and nuclear Nrf2, NQO-1, and HO-1. After UVB stimulation, C-Mc enhanced aggregation of nuclear Nrf2 proteins, which might imply cellular self-preservation (Figure 10(a)). Simultaneously, treatment with C-Mc dramatically increased NQO-1 expression in UVB-exposed NHDFs in a dose- dependent manner, with $67.35 \%$ and $102.14 \%$ increases for $20 \mu \mathrm{M}$ C-Mc (Figure 10(b)).

\section{Discussion}

Interest and research on botanicals as the most promising treatments for skin photoaging have increased in recent years [56]. Because natural active compounds are continually confirmed to have fewer side effects than chemicalbased compounds, increasing numbers of researchers have attempted to extract functionalized materials from naturally occurring substances such as flowers, seeds, roots, and essential oils for use in cosmetics [57]. Medicinal Panax herbs especially Panax quinquefolius L. have been identified as a plant with wide-ranging effects and have been used as a powerful skin antiaging agent [10]. In particular, many studies have assessed for skin antiaging activity of ginsenosides, the principal active components of American ginseng [58]. The major ginsenosides, Rd, Rg1, and Rc, are glycosylated ginsenosides that are poorly absorbed into the gastrointestinal tract. In contrast, minor ginsenosides present in deglycosylated states are relatively easily absorbed into the bloodstream but account for less than 1\% of total ginseng [59]. Owing to the difficulty of extracting and separating of the minor ginsenosides, the identification of the activity of medicament remains a challenge, which hampers their use as drug candidates or cosmetics ingredients.

In this study, we proposed a novel in silico framework for the identification of active ginsenosides and exploration of underlying mechanisms against photoaging. The drug virtual screening framework consists of the following steps: (1) preliminary identification based on shortest distance calculation in the chemical space, (2) candidates prioritizing through pairwise chemical structure similarity analysis, and (3) drug-likeness evaluation and ADMET prediction for the candidates (Supplementary Figure S1). We highlighted C-Mc (20-O-[ $\alpha$-L-arabinofuranosyl- $(1 \rightarrow 6)-\beta$-Dglucopyranosyl]-20(S)-protopanaxdiol), a rare minor ginsenoside, as one of the most promising candidates for the prevention and treatment of photoaging. We further obtained the minor ginsenoside $\mathrm{C}$-Mc through the $\mathrm{Rc} \rightarrow \mathrm{C}$ $\mathrm{Mcl} \rightarrow \mathrm{C}-\mathrm{Mc} \rightarrow \mathrm{C}-\mathrm{K} \quad$ biotransformation pathway and validated its pharmacologic effects of antiphotoaging in UVB-irradiated NHDFs.

System pharmacology analysis was conducted for the exploration of the molecular mechanisms of ginsenosides $\mathrm{C}-\mathrm{Mc}$ against photoaging. We constructed drug-target network of $\mathrm{C}-\mathrm{Mc}$, photoaging-related gene network, and skin tissue-specific expression protein network and performed network analysis and gene enrichment. We predicted four major functional modules of $\mathrm{C}-\mathrm{Mc}$ against photoaging, including inflammation, collagen synthesis and metabolic, secretion of MMPs, and oxidative stress, which are intrinsically connected. In vitro experiments confirmed these hypotheses and further elucidated the cytoprotective mechanism of actions for C-Mc, as summarized in Figure 11.

UVB is the primary external factor that induces ROS, which in turn stimulate many cascades and ultimately lead to premature skin senescence [60]. We demonstrated that 
ginsenoside C-Mc prevented ROS production (Figures 4(b)$4(\mathrm{~d})$ ) and decreased MMP secretion (Figures 7(a)-7(d)) caused by UVB irradiation and showed a protective action against photooxidative aging. Furthermore, C-Mc effectively eliminated UVB-exposed ROS generation and upregulated expression of the antioxidant enzymes HO-1 and NQO-1 by facilitating translocation of $\mathrm{Nrf} 2$ from the cytoplasm to the nucleus (Figure 10). These data indicate that C-Mc has antioxidant properties by increasing levels of key endogenous cutaneous antioxidant factors.

UV radiation activates an intricate cascade of biochemical reactions in human skin. UV radiation is known to be a strong activator of skin NF- $\kappa$ B via suppression of $\mathrm{I} \kappa \mathrm{B}-\alpha$ protein synthesis [61]. Expression of NF- $\kappa$ B stimulates the release of proinflammatory cytokines such as TNF- $\alpha$, iNOS, and IL-6 [62]. Previous research showed that TNF- $\alpha$ and IL6 inhibit the production of collagen, and iNOS dramatically suppresses ECM synthesis by reducing the expression of type I collagen [63]. Consistent with previously published studies, we showed that C-Mc significantly inhibited the expression of IL-6, iNOS, and TNF- $\alpha$ (Figures $8(a)$ and $8(\mathrm{c})-8(\mathrm{e})$ ), increased TGF- $\beta 1$ secretion (Figures 9(b) and 9(d)), and upregulated procollagen type I (Figures 9(a) and 9(c)) expression to facilitate collagen synthesis in a concentration-dependent manner. In addition, molecular dynamics simulation manifested C-Mc has potentially therapeutic photoaging effect by suppressing MAPK, NF- $\kappa \mathrm{B}$, IL6 , and TNF- $\alpha$ (Supplementary Figure S2 and Table S2). The MAPK/NF- $\kappa$ B pathway stimulates expression of inflammation-related genes and is associated with the MMP overexpression induced by UVB irradiation, especially MMP-1 [64]. Use of the MAPK inhibitors PD98950 (an ERK inhibitor) and SP600125 (a JNK inhibitor) revealed relationships between MMP-1, IL-6, and the MAPK pathway in the antiaging mechanism of CMc (Supplementary Figure S5). As expected, the MAPK inhibitors PD98950 and SP600125 decreased expression of MMP-1 and IL-6, indicating that ginsenoside C-Mc inhibits expression of these proteins through regulation of the MAPK signaling pathway. Moreover, C-Mc scavenged ROS (Figures $4(\mathrm{~b})-4(\mathrm{~d})$ ), as illustrated by reduced LDH and increased intracellular GSH expression levels in UVBexposed NHDFs (Figures 4(f) and 4(g)). This finding suggests that the antiphotoaging effects of $\mathrm{C}-\mathrm{Mc}$ are due to activation or induction of antioxidant constituents that can reduce the overproduction of ROS. In general, we hypothesize that $\mathrm{C}-\mathrm{Mc}$ is an excellent natural antiinflammatory and antioxidant product for photodamaged skin.

As the precursor of collagen type I, procollagen type I is important for maintaining skin flexibility and resilience [65]. Repetitive or acute exposure to UV irradiation stimulates excessive MMP-1 secretion, which a significant cause of loss of type I collagen in the dermal ECM and alters the physical properties of skin, leading to primary skin aging [66]. UV radiation inhibits procollagen type I synthesis through the TGF- $\beta /$ Smad pathway [67]. Consistent with these findings, C-Mc showed bioactivity associated with upregulation of TGF- $\beta 1$, enhanced phosphorylation of Smad2/3 expression, and remarkably reduced Smad7 expression level in UVBexposed NHDFs (Figure 9(d)). Therefore, we suggest that regulation of factors associated with the TGF- $\beta /$ Smad pathway might effectively protect cells against UVB-induced photodamage.

Dermatology studies show that VEGF plays a critical role in pathological mechanisms of skin psoriasis and carcinoma [68]. Moreover, VEGF overexpression enhances sensitivity to UVB irradiation while accelerated inflammation expedites photoaging [69]. Broadly speaking, weaken VEGF secretion could be an effective approach to apply to the prevention or treatment of UVB-induced photoaging. Our findings indicated that elevated VEGF secretion resulting from UVB-exposed is conspicuously lowered by treated ginsenoside C-Mc (Figure 8(b)). Hence, mechanisms of UVBexposed alter in VEGF expression deserved for further investigation in the future.

Overall, the results of this study indicated that the in silico approaches proposed here may serve as a novel and effective strategy to accelerate the discovery of antiphotoaging agent from ginsenosides. Yet several shortcomings of the presented study should be acknowledged. Firstly, this study predicted that three rare minor ginsenosides (C-Mc, $\mathrm{Mx}$, and F2) may possess high potential as antiphotoaging agents. Although C-Mc has shown significant antioxidant and cytoprotective activity against UVB-induced photodamage in human dermal fibroblasts, the remaining ginsenosides (Mx and F2) deserved to be further validated by wet-lab assays in the future. Secondly, this study merely preliminary investigated the antiphotoaging effects and mechanisms at the cellular level; further in-depth experiments should be conducted in mice, artificial skin, and human skin to explore the safety and effectiveness of ginsenoside C-Mc as orally or tropically agent.

\section{Conclusion}

This study presented a novel and useful in silico drug discovery strategy and identified the rare minor ginsenoside $\mathrm{C}-\mathrm{Mc}$ as a promising antiphotoaging compound from over 80 ginsenosides. In combination with system pharmacology-based prediction and in vitro validation, we found that C-Mc suppressed MMP production via regulating the MAPK/AP-1/ $\mathrm{NF}-\kappa \mathrm{B}$ pathway as well as expedited collagen synthesis via the TGF- $\beta /$ Smad pathway. In addition, C-Mc enhanced the expression of Nrf2/ARE to hold a balance of endogenous oxidation. We believe that C-Mc will prove to be a useful cosmetic agent and drug candidate, particularly for skin care, and might be used to reduce UVB-induced skin photodamage.

\section{Data Availability}

The original contributions presented in the study are included in the article/supplementary material; further inquiries can be directed to the corresponding authors upon reasonable request. 


\section{Conflicts of Interest}

The authors declare that there is no conflict of interest regarding the publication of this paper.

\section{Authors' Contributions}

CC, TY, and YK conceived and designed the experiments. $\mathrm{CC}$ and XL conducted the experiments and wrote the manuscript. HL and SL performed data collection. EH, BP, YX, and JF participated in the experiments. CC, TY, and YK reviewed the manuscript. $\mathrm{CC}$ and $\mathrm{XL}$ revised the manuscript. All authors have read and approved the final manuscript.

\section{Acknowledgments}

This work was supported by the National Natural Science Foundation of China (No. 81903912), the Science and Technology Plan Project of Guangzhou of China (Grant No. 20210202049), and the STU Scientific Research Foundation for Talents (No. NTF21034). The authors are grateful to the support of Laboratory of TCM Syndrome Essence and Objectification.

\section{Supplementary Materials}

Figure S1: overall workflow of this study. Figure S2: molecular docking mode of the complex 3D structure. Figure S3: chromatographic analysis of ginsenoside C-Mc. Figure S4: cell viability of ginsenoside C-Mc. Figure S5: effects of MAPK inhibitors on UVB-exposed MAPK activation and MMP-1, IL-6 secretion in UVB-irradiated NHDFs. Table S1: information, structures, and antiphotoaging reference of ginsenosides collecting from literatures and PubChem database. Table S2: binding energy and hydrogen bonding interactions between atomic groups of receptor and ligand. Table S3: drug-target network of ginsenoside C-Mc. Table S4: list of the 124 skin photoaging-related genes. Table S5: the skin tissue-specific expression protein network containing 907 proteins. Table S6: real-time PCR primers. Table S7: the 22 ginsenosides with significant shortest distance $\left(D_{\min }<0.2\right)$ to the set of known antiphotoaging ginsenosides. (Supplementary Materials)

\section{References}

[1] A. Han, A. L. Chien, and S. J. Kang, "PhotoagingPhotoaging," Dermatologic clinics, vol. 32, no. 3, pp. 291-299, 2014.

[2] M. Cavinato and P. Jansen-Dürr, "Molecular mechanisms of UVB-induced senescence of dermal fibroblasts and its relevance for photoaging of the human skin," Experimental Gerontology, vol. 94, pp. 78-82, 2017.

[3] A. C. Weihermann, M. Lorencini, C. A. Brohem, and C. M. De Carvalho, "Elastin structure and its involvement in skin photoageing," International journal of cosmetic science, vol. 39, no. 3, pp. 241-247, 2017.

[4] A. Kammeyer and R. M. Luiten, "Oxidation events and skin aging," Ageing research reviews, vol. 21, 2015.
[5] Y. Cheong, C. Kim, M. B. Kim, and J. K. Hwang, "The antiphotoaging and moisturizing effects of Bouea macrophylla extract in UVB-irradiated hairless mice," Food Science and Biotechnology, vol. 27, no. 1, pp. 147-157, 2018.

[6] A. Wiraguna, W. Pangkahila, and I. N. M. Astawa, "Antioxidant properties of topical Caulerpa sp. extract on UVBinduced photoaging in mice," Dermatology reports, vol. 10, no. 2, p. 7597, 2018.

[7] X. Zhai, M. Gong, Y. Peng, and D. Yang, "Effects of UV induced-photoaging on the hair follicle cycle of C57BL6/J mice," Clinical, Cosmetic and Investigational Dermatology, vol. Volume 14, pp. 527-539, 2021.

[8] I. Sjerobabski Masnec and S. Poduje, "Photoaging," Collegium Antropologicum, vol. 32, Suppl 2, pp. 177-180, 2008.

[9] L. Wang, X. Yu, X. Yang, Y. Yang, and G. Ren, "Structural and anti-inflammatory characterization of a novel neutral polysaccharide from North American ginseng (Panax quinquefolius)," International journal of biological macromolecules, vol. 74, 2015.

[10] S. Sabouri-Rad, S. Sabouri-Rad, A. Sahebkar, and Z. TayaraniNajaran, "Ginseng in dermatology: a review," Current Pharmaceutical Design, vol. 23, no. 11, pp. 1649-1666, 2017.

[11] E. K. Park, M. K. Choo, M. J. Han, and D. H. Kim, "ginsenoside Rh1 possesses antiallergic and anti-inflammatory activities," International archives of allergy and immunology, vol. 133, no. 2, pp. 113-120, 2004.

[12] J. Chen, H. Peng, X. Ou-Yang, and X. He, "Research on the antitumor effect of ginsenoside Rg3 in B16 melanoma cells," Melanoma research, vol. 18, no. 5, pp. 322-329, 2008.

[13] Y. Cheng, L. H. Shen, and J. T. Zhang, "Anti-amnestic and anti-aging effects of ginsenoside Rg1 and Rb1 and its mechanism of action," Acta Pharmacologica Sinica, vol. 26, no. 2, pp. 143-149, 2005.

[14] J. Han, E. Lee, E. J. Kim et al., "Role of epidermal $\gamma \delta$ T-cellderived interleukin 13 in the skin-whitening effect of ginsenoside F1," Experimental dermatology, vol. 23, no. 11, pp. 860862, 2014.

[15] G. L. Dong, J. Lee, I. H. Cho et al., "Ginsenoside Rg12, a new dammarane-type triterpene saponin from Panax ginseng root," Journal of ginseng research, vol. 41, no. 4, pp. 531-533, 2017.

[16] X. Y. Liu, Y. K. Xiao, E. Hwang, J. J. Haeng, and T. H. Yi, “Antiphotoaging and antimelanogenesis properties of ginsenoside $\mathrm{C}-\mathrm{Y}$, a ginsenoside $\mathrm{Rb} 2$ metabolite from American ginseng PDD-ginsenoside," Photochemistry and Photobiology, vol. 95, no. 6, pp. 1412-1423, 2019.

[17] X. D. Yang, Y. Y. Yang, D. S. Ouyang, and G. P. Yang, “A review of biotransformation and pharmacology of ginsenoside compound K," Fitoterapia, vol. 100, pp. 208-220, 2015.

[18] H. Lee, Y. Hong, Q. Tran et al., "A new role for the ginsenoside RG3 in antiaging via mitochondria function in ultravioletirradiated human dermal fibroblasts," Journal of ginseng research, vol. 43, no. 3, p. 431, 2019.

[19] X. Y. Liu, E. Hwang, B. Park, H. Ngo, Y. K. Xiao, and T. H. Yi, "Ginsenoside C-Mx isolated from notoginseng stem-leaf ginsenosides attenuates ultraviolet B-mediated photoaging in human dermal fibroblasts," Photochemistry and photobiology, vol. 94, no. 5, pp. 1040-1048, 2018.

[20] C. Cai, Q. Wu, H. Hong et al., "In silico identification of natural products from traditional Chinese medicine for cancer immunotherapy," Scientific Reports, vol. 11, no. 1, p. 3332, 2021. 
[21] Q. Wu, Y. Chen, Y. Gu et al., "Systems pharmacology-based approach to investigate the mechanisms of DangguiShaoyao-san prescription for treatment of Alzheimer's disease," BMC complementary medicine and therapies, vol. 20, no. 1, p. 282, 2020.

[22] D. Li, C. Cai, Y. Liao et al., "Systems pharmacology approach uncovers the therapeutic mechanism of medicarpin against scopolamine-induced memory loss," Phytomedicine: international journal of phytotherapy and phytopharmacology, vol. 91, p. 153662, 2021.

[23] C. Cai, Q. Wu, Y. Luo et al., "In silico prediction of ROCK II inhibitors by different classification approaches," Molecular Diversity, vol. 21, no. 4, pp. 791-807, 2017.

[24] C. Cai, P. Guo, Y. Zhou et al., "Deep learning-based prediction of drug-induced cardiotoxicity," Journal of Chemical Information and Modeling, vol. 59, no. 3, pp. 1073-1084, 2019.

[25] Q. Wu, C. Cai, P. Guo et al., "In silico identification and mechanism exploration of hepatotoxic ingredients in traditional Chinese medicine," Frontiers in Pharmacology, vol. 10, p. 458, 2019.

[26] J. Fang, G. Li, M. Huili et al., "Quantitative and systems pharmacology 3. Network-based identification of new targets for natural products enables potential uses in aging-associated disorders," Frontiers in Pharmacology, vol. 8, p. 747, 2017.

[27] J. Fang, C. Cai, Y. Chai et al., "Quantitative and systems pharmacology 4. Network-based analysis of drug pleiotropy on coronary artery disease," European Journal of Medicinal Chemistry, vol. 161, pp. 192-204, 2019.

[28] C. Cai, L. Xu, J. Fang et al., "In silico prediction and bioactivity evaluation of chemical ingredients against influenza a virus from Isatis tinctoria L," Frontiers in Pharmacology, vol. 12, article 755396, 2021.

[29] N. M. O'Boyle, M. Banck, C. A. James, C. Morley, T. Vandermeersch, and G. R. Hutchison, "Open babel: an open chemical toolbox," Journal of Cheminformatics, vol. 3, no. 1, p. 33, 2011.

[30] S. Vilar, G. Cozza, and S. Moro, "Medicinal chemistry and the molecular operating environment (MOE): application of QSAR and molecular docking to drug discovery," Current topics in medicinal chemistry, vol. 8, no. 18, pp. 1555-1572, 2008.

[31] H. Abdi and L. Williams, "Principal component analysis," Computational Statistics, vol. 2, no. 4, pp. 433-459, 2010.

[32] J. Dong, N. N. Wang, Z. J. Yao et al., “ADMETlab: a platform for systematic ADMET evaluation based on a comprehensively collected ADMET database," Journal of Cheminformatics, vol. 10, no. 1, p. 29, 2018.

[33] J. B. Baell and G. A. Holloway, "New substructure filters for removal of pan assay interference compounds (PAINS) from screening libraries and for their exclusion in bioassays," Journal of Medicinal Chemistry, vol. 53, no. 7, pp. 2719-2740, 2010.

[34] G. Xiong, Z. Wu, J. Yi et al., “ADMETlab 2.0: an integrated online platform for accurate and comprehensive predictions of ADMET properties," Nucleic Acids Research, vol. 49, no. W1, pp. W5-w14, 2021.

[35] H. Y. Noh, J. Lu, M. H. Siddiqi et al., "Computational investigation of ginsenoside $\mathrm{F} 1$ from $<\mathrm{i}>$ Panax ginseng $<$ i $>$ meyer as p38 MAP kinase inhibitor: molecular docking and dynamics simulations, ADMET analysis, and drug likeness prediction," Iranian journal of pharmaceutical research: IJPR, vol. 17, no. 4, pp. 1318-1327, 2018.
[36] G. M. Morris, R. Huey, W. Lindstrom et al., “AutoDock4 and AutoDockTools4: automated docking with selective receptor flexibility," Journal of Computational Chemistry, vol. 30, no. 16, pp. 2785-2791, 2009.

[37] Z. Liu, C. Cai, J. Du et al., "TCMIO: a comprehensive database of traditional Chinese medicine on immuno-oncology," Frontiers in Pharmacology, vol. 11, p. 439, 2020.

[38] Z. Wu, Y. Peng, Z. Yu, W. Li, G. Liu, and Y. Tang, "NetInfer: a web server for prediction of targets and therapeutic and adverse effects via network-based inference methods," Journal of Chemical Information and Modeling, vol. 60, no. 8, pp. 3687-3691, 2020.

[39] J. Fang, Z. Wu, C. Cai, Q. Wang, Y. Tang, and F. Cheng, "Quantitative and systems pharmacology. 1. In silico prediction of drug-target interactions of natural products enables new targeted cancer therapy," Journal of Chemical Information and Modeling, vol. 57, no. 11, pp. 2657-2671, 2017.

[40] B. A. Cho, S. K. Yoo, and J. S. Seo, "Signatures of photo-aging and intrinsic aging in skin were revealed by transcriptome network analysis," Aging, vol. 10, no. 7, pp. 1609-1626, 2018.

[41] W. Yan, L. Zhang, S. Liu et al., "Screening of genes associated with skin photoaging by microarray analysis," Chinese Journal of Dermatology, vol. 44, no. 2, pp. 130-133, 2011.

[42] C. Y. Liu, R. X. Zhou, C. K. Sun et al., "Preparation of minor ginsenosides $\mathrm{C}-\mathrm{Mc}, \mathrm{C}-\mathrm{Y}, \mathrm{F} 2$, and $\mathrm{C}-\mathrm{K}$ from American ginseng PPD-ginsenoside using special ginsenosidase type-I from Aspergillus niger g.848," Journal of ginseng research, vol. 39, no. 3, pp. 221-229, 2015.

[43] C. Cai, J. Fang, P. Guo et al., "In silico pharmacoepidemiologic evaluation of drug-induced cardiovascular complications using combined classifiers," Journal of Chemical Information and Modeling, vol. 58, no. 5, pp. 943-956, 2018.

[44] Q. Wu, X. Fan, H. Hong et al., "Comprehensive assessment of side effects in COVID-19 drug pipeline from a network perspective," Food and Chemical Toxicology: An International Journal Published for the British Industrial Biological Research Association, vol. 145, article 111767, 2020.

[45] J. Y. Wan, Y. Fan, Q. T. Yu et al., "Integrated evaluation of malonyl ginsenosides, amino acids and polysaccharides in fresh and processed ginseng," Journal of pharmaceutical and biomedical analysis, vol. 107, pp. 89-97, 2015.

[46] X. Wang, T. Sakuma, E. Asafu-Adjaye, and G. K. Shiu, "Determination of ginsenosides in plant extracts from Panax ginseng and Panax quinquefolius L. by LC/MS/MS," Analytical Chemistry, vol. 71, no. 8, pp. 1579-1584, 1999.

[47] Y. Oh, H. W. Lim, Y. H. Huang et al., “Attenuating properties of Agastache rugosa leaf extract against ultraviolet-B-induced photoaging via up-regulating glutathione and superoxide dismutase in a human keratinocyte cell line," Journal of Photochemistry and Photobiology B: Biology, vol. 163, pp. 170-176, 2016.

[48] G. Bindea, B. Mlecnik, H. Hackl et al., "ClueGO: a Cytoscape plug-in to decipher functionally grouped gene ontology and pathway annotation networks," Bioinformatics, vol. 25, no. 8, pp. 1091-1093, 2009.

[49] H. S. Han, J. S. Shin, D. B. Myung et al., "Hydrangea serrata (Thunb.) Ser. extract attenuate UVB-induced photoaging through MAPK/AP-1 inactivation in human skin fibroblasts and hairless mice," Nutrients, vol. 11, no. 3, p. 533, 2019.

[50] N. Yoshizaki, T. Fujii, R. Hashizume, and H. Masaki, "A polymethoxyflavone mixture, extracted from orange peels, 
suppresses the UVB-induced expression of MMP-1," Experimental dermatology, vol. 25, Suppl.3, pp. 52-56, 2016.

[51] S. P. Divya, X. Wang, P. Pratheeshkumar et al., "Blackberry extract inhibits UVB-induced oxidative damage and inflammation through MAP kinases and NF- $\kappa \mathrm{B}$ signaling pathways in SKH-1 mice skin," Toxicology and applied pharmacology, vol. 284, no. 1, pp. 92-99, 2015.

[52] T. Chen and H. Hou, "Protective effect of gelatin polypeptides from Pacific cod (Gadus macrocephalus) against UV irradiation-induced damages by inhibiting inflammation and improving transforming growth factor- $\beta /$ Smad signaling pathway," Journal of Photochemistry and Photobiology B: Biology, vol. 162, pp. 633-640, 2016.

[53] T. L. Cezar, R. M. Martinez, C. da Rocha et al., "Treatment with maresin 1, a docosahexaenoic acid-derived proresolution lipid, protects skin from inflammation and oxidative stress caused by UVB irradiation," Scientific reports, vol. 9, no. 1, p. 3062, 2019.

[54] C. Ji, J. W. Huang, Q. Y. Xu, Z. Jing, and C. J. Bo, “Gremlin inhibits UV-induced skin cell damages via activating VEGFR2-Nrf2 signaling," Oncotarget, vol. 7, no. 51, p. 84748, 2016.

[55] P. Y. Wu, L. Jia-Ling, Y. J. Liu et al., "Fisetin regulates Nrf2 expression and the inflammation-related signaling pathway to prevent UVB-induced skin damage in hairless mice," International journal of molecular sciences, vol. 18, no. 10, p. 2118, 2017.

[56] M. Cavinato, B. Waltenberger, G. Baraldo, C. V. Grade, H. Stuppner, and P. Jansen-Dürr, "Plant extracts and natural compounds used against UVB-induced photoaging," Biogerontology, vol. 18, no. 4, pp. 499-516, 2017.

[57] N. Saewan and A. Jimtaisong, "Natural products as photoprotection," Journal of cosmetic dermatology, vol. 14, no. 1, pp. 4763, 2015.

[58] T. G. Lim, C. C. Lee, Z. Dong, and K. W. Lee, "Ginsenosides and their metabolites: a review of their pharmacological activities in the skin," Archives of dermatological research, vol. 307, p. 5, 2015.

[59] X. J. Lei, K. Feng, L. W. Sun, R. Jiang, L. I. Chang-Yu, and Y. P. Wang, "Progress in studying anti-aging mechanism of ginsenoside," Amino Acids \& Biotic Resources, vol. 1, 2010.

[60] S. Kawashima, T. Funakoshi, Y. Sato et al., "Protective effect of pre- and post-vitamin C treatments on UVB-irradiationinduced skin damage," Scientific reports, vol. 8, no. 1, 2018.

[61] S. Lalita, L. T. Hwan, W. H. Mustatab, B. So-Hyeon, and K. S. Yeou, "Resveratrol-enriched rice attenuates UVB-ROSinduced skin aging via downregulation of inflammatory cascades," Oxidative Medicine and Cellular Longevity, vol. 2017, Article ID 8379539, 15 pages, 2017.

[62] S. Joy, K. Cheol-Su, K. Soo-Ki, S. K. Yong, T. Y. Kang, and L. Kyu-Jae, "Antioxidant and anti-inflammatory effects of shungite against ultraviolet B irradiation-induced skin damage in hairless mice," Oxidative medicine and cellular longevity, vol. 2017, 11 pages, 2017.

[63] F. Verre Cc Hia and A. Mauviel, "TGF-beta and TNF-alpha: antagonistic cytokines controlling type I collagen gene expression," Cellular signalling, vol. 16, no. 8, pp. 873-880, 2004.
[64] M. M. Oliveira, B. A. Ratti, R. G. Daré et al., "Dihydrocaffeic acid prevents UVB-induced oxidative stress leading to the inhibition of apoptosis and MMP-1 expression via p38 signaling pathway," Oxidative Medicine and Cellular Longevity, vol. 2019, Article ID 2419096, 14 pages, 2019.

[65] J. Varani, D. Spearman, P. Perone et al., "Inhibition of type I procollagen synthesis by damaged collagen in photoaged skin and by collagenase-degraded collagen in vitro," The American journal of pathology, vol. 158, no. 3, pp. 931-942, 2001.

[66] C. Chiu-Lan, L. Shu-Fen, C. Su-Jong, and S. Mei-Fen, "Protective effects of Chlorella-derived peptide on UVB-induced production of MMP-1 and degradation of procollagen genes in human skin fibroblasts," Regulatory toxicology and pharmacology, vol. 60, no. 1, pp. 112-119, 2011.

[67] J. Massagué and D. Wotton, "NEW EMBO MEMBERS REVIEW: Transcriptional control by the TGF-beta/Smad signaling system," The EMBO journal, vol. 19, no. 8, pp. 17451754, 2000.

[68] S. G. Almalki and D. K. Agrawal, "ERK signaling is required for VEGF-A/VEGFR2-induced differentiation of porcine adipose-derived mesenchymal stem cells into endothelial cells," Stem cell research \& therapy, vol. 8, no. 1, p. 113, 2017.

[69] H. Eunson, P. Lin, H. Ngo et al., "Icariin and icaritin recover UVB-induced photoaging by stimulating Nrf2/ARE and reducing $\mathrm{AP}-1$ and $\mathrm{NF}-\kappa \mathrm{B}$ signaling pathways: a comparative study on UVB-irradiated human keratinocytes," Photochemical \& Photobiological Sciences, vol. 17, no. 10, pp. 1396-1408, 2018. 